\title{
On Local Congruence of Immersions in Homogeneous or Nonhomogeneous Spaces
}

Jeongoo CHEH

Department of Mathematics 85 Statistics, The University of Toledo, Toledo, OH 43606, USA

E-mail: jeongoocheh@gmail.com

Received May 14, 2012, in final form April 19, 2013; Published online April 28, 2013

http://dx.doi.org/10.3842/SIGMA.2013.036

\begin{abstract}
We show how to find a complete set of necessary and sufficient conditions that solve the fixed-parameter local congruence problem of immersions in $G$-spaces, whether homogeneous or not, provided that a certain $k^{\text {th }}$ order jet bundle over the $G$-space admits a $G$-invariant local coframe field of constant structure. As a corollary, we note that the differential order of a minimal complete set of congruence invariants is bounded by $k+1$. We demonstrate the method by rediscovering the speed and curvature invariants of Euclidean planar curves, the Schwarzian derivative of holomorphic immersions in the complex projective line, and equivalents of the first and second fundamental forms of surfaces in $\mathbb{R}^{3}$ subject to rotations.
\end{abstract}

Key words: congruence; nonhomogeneous space; equivariant moving frame; constantstructure invariant coframe field

2010 Mathematics Subject Classification: 53A55; 53B25

Dedicated to my teacher, Professor Peter Olver, in honor of his sixtieth birthday.

\section{Introduction}

The equivalence problem of immersed submanifolds of a manifold $M$ is to find a set of computable criteria that determine whether the submanifolds, or open subsets thereof in their own topology, are equivalent or not under the action of a prescribed symmetry group ${ }^{1} G$ of $M$. Recall that a manifold $M$ on which a (local) Lie group $G$ acts is called a $G$-space. For a homogeneous $G$-space $M$, there is a classical solution to the equivalence problem, known as Cartan's moving frame method, $[4,5,7,15]$, that consists of lifting the submanifolds into the principal $H$-bundle $G$ over $M$, where $H<G$ is the stabilizer of a point of $M$, to pull the Maurer-Cartan forms of $G$ back down to the submanifolds and then use an argument resembling Cartan's technique of the graph, [16], that is Cartan's rendition of the Frobenius theorem.

To our knowledge, however, little has been known of any general solution for the case of submanifolds of nonhomogeneous spaces. One of the obvious obstacles in one's attempt to apply the classical method to the case of a nonhomogeneous space $M$ is the unavailability of any natural manifestation of $G$ as a principal bundle over $M$, unlike in the case of homogeneous spaces.

In $[2,3]$, remarks (for example, Theorem 7.2 in [3]) have been made on a solution to a certain type of equivalence problems of submanifolds of a space where its symmetry group may not act transitively. Its method involves using an extended invariant coframe field that consists of an

${ }^{\star}$ This paper is a contribution to the Special Issue "Symmetries of Differential Equations: Frames, Invariants and Applications". The full collection is available at http://www.emis.de/journals/SIGMA/SDE2012.html

${ }^{1}$ The symmetry group of a space is by definition the group of all admissible transformations that are typically characterized to preserve, for instance, certain geometric structures on the space; conversely, prescribing a symmetry group to a space amounts to declaring the class of admissible transformations of the space. 
invariant coframe field along with a complete system of differential invariants. Although the papers dealt with equivalence problems somewhat different ${ }^{2}$ from those that we will study in our own paper, they have stimulated our curiosity and motivation by drawing our attention to the article [5] that studied equivalence problems for submanifolds immersed exclusively in homogeneous spaces.

Besides the issue of whether the ambient space is homogeneous or nonhomogeneous, there is another technicality related to reparametrization of submanifolds. Suppose that $\psi_{1}, \psi_{2}: X \longrightarrow M$ are two submanifolds of a $G$-space $M$. One type of equivalence problem is to determine whether there exits an element $g \in G$ that transforms one image set $\psi_{1}(X)$ to the other image set $\psi_{2}(X)$, i.e., $g\left(\psi_{1}(X)\right)=\psi_{2}(X)$. Since this problem is concerned only with the images of the submanifold immersions, reparametrizing a submanifold, such as $\psi_{1} \circ \phi: X \longrightarrow M$ with a (local) diffeomorphism $\phi: X \longrightarrow X$, should not change the final answer of the equivalence problem.

The equivalence problem allowing reparametrization of submanifolds has been studied extensively, for instance, in [11]. The overall idea of the solution for this problem largely consists of two parts. First, find a (minimal) set of differential invariants that generate, through invariant differentiation, the entire algebra of differential invariants. Then restrict the differential invariants of up to a certain enough order to the given submanifolds to obtain signature submanifolds, which in turn leads to the final question on overlapping submanifolds. We should note the theoretical development in a novel and systematic method of finding a generating set of differential invariants under finite- or infinite-dimensional Lie pseudo-group actions, [13, 14], and its applications to a number of different geometries, [6, 10, 12].

The other type of equivalence problem is concerned with not only the images of the immersions but also the immersions themselves as well. In other words, even when the images may be regarded equivalent in the previous sense (that we call variable-parameter congruence) of allowing reparametrization of immersed submanifolds, if one of the submanifolds is indeed nontrivially reparametrized, the immersed submanifolds are no longer to be considered equivalent in the new sense. It is this type of more restrictive (and hence easier) equivalence problem that we will study in our paper, and we call it the fixed-parameter congruence problem. The precise meaning of congruence is as follows:

Definition 1.1. Suppose that $\psi_{1}, \psi_{2}: X \longrightarrow M$ are immersions of a manifold $X$ into a $G$ space $M$. We say that $\psi_{1}$ and $\psi_{2}$ are congruent at $x_{0} \in X$ if there exist an open neighborhood $U \subset X$ of $x_{0}$ and a transformation $g \in G$ such that $\psi_{1}(x)=g \circ \psi_{2}(x)$ for all $x \in U$.

The main goal of our paper is to provide a theoretical justification of a method that we devised for solving the local congruence problem of immersed submanifolds of a $G$-space that may or may not be homogeneous. In essence, our method is a hybrid of the classical Cartan's method, [4, 5, 7, 15], and the method of invariant coframe fields constructed by equivariant moving frames, $[2,3,8,14]$. On the one hand, we follow the classical idea of utilizing Cartan's technique of the graph which provides a means to solve the equivalence problem by examining certain invariant coframe fields; on the other hand, we use the machinery of equivariant moving frames, as developed by Olver et al., to construct particular invariant coframe fields that are of constant structure (Definition 4.2). One of our main results is Theorem 4.3 where we give a proof to the effect that we can use an invariant coframe field of constant structure, instead of the unavailable Maurer-Cartan forms of homogeneous spaces required by the classical method, to completely determine congruence of immersions whether the ambient space is homogeneous or not. We regard our result as extending and generalizing to quite arbitrary $G$-spaces the key lemmas proved in [5] that solved congruence problems in homogeneous spaces. A notable

\footnotetext{
${ }^{2}$ Shortly, we will explain two different types of equivalence problems: the variable-parameter and fixedparameter types.
} 
corollary to Theorem 4.3 is that if the prolongation of the (verticalized ${ }^{3}$ ) action of the group becomes locally free at order $k$, then a minimal complete set of congruence invariants should be of order $k+1$ or less.

We illustrate our method by finding congruence conditions of some specific well-known examples, which will thus help attest to the validity of our method. Through the first few sections of the paper, we explain key ideas of our method with the example of the congruence problem of curves in $\mathbb{R}^{2}$ under the action of the orientation-preserving rigid motion group $\mathrm{SE}(2)$, whereby we obtain speed and curvature congruence invariants of curves. The last section of the paper focuses on three examples. We rediscover the Schwarzian derivative as a congruence invariant of holomorphic immersions, or biholomorphisms, in the complex projective line $\mathbb{C P}^{1}$ under the action of the projective special linear group $\operatorname{PSL}(2, \mathbb{C})$ or alternatively in the complex plane $\mathbb{C}$ under the action of linear fractional, or Möbius, transformations. The next example obtains a complete system of congruence invariants, including equivalents to the first and second fundamental forms of surfaces in $\mathbb{R}^{3}$, under the intransitive action of the rotation group $\mathrm{SO}(3)$. The last example computes congruence conditions for surfaces in $\mathbb{R}^{3}$ under another intransitive action of a certain subgroup of a Heisenberg group.

Throughout the paper, all manifolds and maps are assumed to be smooth, $M$ always denotes a smooth manifold, and $G$ an $r$-dimensional local Lie group acting on $M$ and other manifolds. Furthermore, since we are concerned only with local equivalence, by a manifold will we actually mean a connected open subset thereof that is small enough to suit the context, and likewise by $G$ a sufficiently small connected open neighborhood of its identity element. However, whenever serious confusion may be possible, we will explicitly remind ourselves of the local nature of various spaces and maps involved.

\section{Verticalization and prolongation of group actions}

To study the effects of group actions on immersed submanifolds of $M$, we begin by treating the submanifolds as (local) sections of the product bundle $X \times M \longrightarrow X$ where $X$ denotes the space of parameters of the submanifolds or the domain of the maps immersing the submanifolds. This viewpoint establishes a one-to-one correspondence $\gamma$ between the set of immersions $f: X \longrightarrow M$ and the set of sections $\gamma(f)$ defined by

$$
\gamma(f): X \longrightarrow X \times M, \quad \gamma(f)(x)=(x, f(x)) .
$$

The action of $G$ on $M$ induces an obvious natural action on $X \times M$ :

Definition 2.1. The verticalized action of $G$ is defined to be

$$
G \times(X \times M) \longrightarrow X \times M, \quad(g,(x, u)) \longmapsto(x, g \cdot u) .
$$

It is this action of $G$ on $X \times M$ that we will later prolong to the jet bundles $J^{k}(X, M)$, $k=0,1,2, \ldots$, of local sections of the bundle $X \times M \longrightarrow X$.

Remark 2.2. In the literature, if $M$ itself is a fibered manifold, it is customary, for the sake of computation in local coordinates, to consider the jet bundles $J^{k} M$ of only those submanifolds of $M$ that are transverse to the fibers of $M$. Our construction of the bundles $J^{k}(X, M)$, on the other hand, contains prolongations, [9, 11], of all submanifolds of $M$, including even those that may not be transverse to the fibers of $M$. Furthermore, the local coordinate description of the prolonged action of $G$ on $J^{k}(X, M)$ turns out much simpler than that on $J^{k} M$ since the verticalized action of $G$ on $X \times M$ does not affect base $(X)$ coordinates at all.

\footnotetext{
${ }^{3}$ See Definition 2.1 .
} 
Let $\left(x^{1}, \ldots, x^{p}\right)$ and $\left(u^{1}, \ldots, u^{n}\right)$ denote local coordinate systems on manifolds $X$ and $M$, respectively. We write $\left(x^{1}, \ldots, x^{p}, u^{1}, \ldots, u^{n}, \ldots, u_{J}^{\alpha}, \ldots\right)$, where $\alpha=1,2, \ldots, n$ and $J$ is a symmetric multi-index over $\{1,2, \ldots, p\}$, for the standard bundle-adapted local coordinate system on $J^{k}(X, M)$. Then, for $g \in G$ and $z \in J^{k}(X, M)$, while the base coordinates of $g \cdot z$ remain unchanged:

$$
x^{i}(g \cdot z)=x^{i}(z), \quad i=1,2, \ldots, p,
$$

all the fiber coordinates $u_{J}^{\alpha}(g \cdot z)$ are found by iterating the recursive formula

$$
u_{J, i}^{\alpha}(g \cdot z)=D_{i}\left(u_{J}^{\alpha}(g \cdot z)\right), \quad \alpha=1,2, \ldots, n, \quad i=1,2, \ldots, p,
$$

where

$$
D_{i}:=\frac{\partial}{\partial x^{i}}+\sum_{|J| \geq 0} \sum_{\alpha=1}^{n} u_{J, i}^{\alpha} \frac{\partial}{\partial u_{J}^{\alpha}}, \quad i=1,2, \ldots, p,
$$

are the coordinate-wise total differential operators on the infinite-order Jet bundle $J^{\infty}(X, M)$. For more details on calculus on jet bundles and related notations, refer, for example, to the resources $[1,9,11]$.

Example 2.3 (Euclidean plane). Consider the Euclidean plane $\left(\mathbb{R}^{2}, \mathrm{SE}(2)\right)$ under the transitive action of the group $\mathrm{SE}(2):=\mathrm{SO}(2) \ltimes \mathbb{R}^{2}$ of orientation-preserving rigid motions. Let $\theta \in \mathbb{R}$ and $(a, b) \in \mathbb{R}^{2}$ be the parameters of $\operatorname{SE}(2)$ such that, for $(u, v) \in \mathbb{R}^{2}$, the action is given by

$$
(\theta, a, b) \cdot(u, v) \longmapsto(\widehat{u}, \widehat{v}),
$$

where

$$
\widehat{u}=u \cos \theta-v \sin \theta+a, \quad \widehat{v}=u \sin \theta+v \cos \theta+b .
$$

Let $x$ and $(u, v)$ denote the local coordinate systems on $\mathbb{R}$ and $\mathbb{R}^{2}$, respectively. Then the jet bundle $J^{1}\left(\mathbb{R}, \mathbb{R}^{2}\right)$ of local sections $\gamma(\psi)$ of the product bundle $\mathbb{R} \times \mathbb{R}^{2} \longrightarrow \mathbb{R}$, where $\psi: \mathbb{R} \longrightarrow \mathbb{R}^{2}$ is a locally defined immersion, has the standard local coordinate system $\left(x, u, v, u_{x}, v_{x}\right)$. In these coordinates, the verticalized action of $\mathrm{SE}(2)$ on $\mathbb{R} \times \mathbb{R}^{2}=J^{0}\left(\mathbb{R}, \mathbb{R}^{2}\right)$ is given by the rule

$$
(\theta, a, b) \cdot(x, u, v) \longmapsto(x, \widehat{u}, \widehat{v})
$$

and its prolonged action $\mathcal{A}: \mathrm{SE}(2) \times J^{1}\left(\mathbb{R}, \mathbb{R}^{2}\right) \longrightarrow J^{1}\left(\mathbb{R}, \mathbb{R}^{2}\right)$ by

$$
(\theta, a, b) \cdot\left(x, u, v, u_{x}, v_{x}\right) \longmapsto\left(x, \widehat{u}, \widehat{v}, \widehat{u_{x}}, \widehat{v_{x}}\right)
$$

where, according to (1),

$$
\widehat{u_{x}}=D_{x} \widehat{u}=u_{x} \cos \theta-v_{x} \sin \theta, \quad \widehat{v_{x}}=D_{x} \widehat{v}=u_{x} \sin \theta+v_{x} \cos \theta .
$$

\section{Review of equivariant moving frames}

The purpose of this section is to review and use equivariant moving frames as part of the tools that we will need later on to solve congruence problems. Our main references are $[2,3]$, and we will provide proofs for all our claims whenever they do not have exact counterparts proved in the references.

In general, two special types of maps between $G$-spaces stand out in the business of constructing invariant differential forms. Let $\psi: M \longrightarrow N$ be a locally defined map between two $G$-spaces $M$ and $N$. 
Definition 3.1. Let $V \subset G$ be an open neighborhood of the identity element of $G$. The map $\psi$ is said to be $G$-invariant if

$$
g^{*} \psi:=\psi \circ g=\psi
$$

for all $g \in V$ such that $\psi \circ g$ is well-defined; and $G$-equivariant if

$$
\psi(g \cdot x)=g \cdot \psi(x)
$$

for all $g \in V$ and $x \in M$ such that the both sides of the equation (2) are well defined.

Remark 3.2. Throughout the paper, all our constructions, without exception, of maps, differential forms, group actions, etc. will be done locally. To avoid the trite use of the word local and relevant open subset notations, we henceforth impose the blanket assumption that all domains of maps should be understood to be freely replaceable by appropriately small open subsets thereof in order to make sense of the expressions in which they are considered. Thus, for example, we will simply say that a map $\psi$ is $G$-equivariant instead of more precisely saying that $\psi$ is $V$-equivariant for an open neighborhood $V \subset G$ of the identity element of $G$, and this is what is reflected in Definition 3.1.

Proposition 3.3. If $\psi$ is $G$-invariant and $\omega$ is any differential form on $N$, then $\psi^{*} \omega$ is a $G$ invariant differential form on $M$. On the other hand, if $\psi$ is $G$-equivariant and $\omega$ is a $G$-invariant differential form on $N$, then $\psi^{*} \omega$ is a $G$-invariant differential form on $M$.

Proof. If $\psi: M \longrightarrow N$ is $G$-invariant, then for any $g \in G$ and any differential form $\omega$ on $N$, $g^{*}\left(\psi^{*} \omega\right)=(\psi \circ g)^{*} \omega=\psi^{*} \omega=\omega$, showing that $\psi^{*} \omega$ is $G$-invariant. The proof for the case of equivariant maps is similar.

Thus, when $N$ is the Lie group $G$ that always comes with a canonical family of invariant differential forms, (that is, Maurer-Cartan forms), a $G$-equivariant map $M \longrightarrow G$ plays a key role in producing invariant differential forms on $M$, and thus has acquired a special recognition in [3].

Definition 3.4. Under an action of $G$ on itself, a locally defined $G$-equivariant map $\rho: M \longrightarrow G$, is called an (equivariant) moving frame. Associated to $\rho$ is the locally defined (equivariant) moving frame section

$$
\sigma: M \longrightarrow G \times M, \quad z \longmapsto(\rho(z), z),
$$

of the trivial product bundle $G \times M \longrightarrow M$.

When the $G$-action on $M$ is locally free, a typical procedure for obtaining a moving frame begins by choosing a local cross-section $\Gamma$ transverse to the $G$-orbits in $M$ and then defining a map $\rho: M \longrightarrow G$ by requiring $\rho(z) \cdot z \in \Gamma$ for $z \in M$. If the $G$-action on $M$ is a left action ${ }^{4}$ and if $G$ acts on itself by the rule $g \cdot h \longmapsto h g^{-1}$, then the map $\rho$ defined in such a way is indeed a moving frame satisfying the condition: for all $z \in M$ and $g \in G, \rho(g \cdot z)=\rho(z) g^{-1}$ whenever the two sides are well defined. If we have a right $G$-action on $M$ and if we define the right action of $G$ on itself by $g \cdot h \longmapsto g^{-1} h$, then the map $\rho$ constructed above is again a moving frame, this time satisfying $\rho(g \cdot z)=g^{-1} \rho(z)$.

As long as $G$ acts on itself locally freely (for example, via left or right translation), local freeness of the group action on $M$ is also a necessary condition for the existence of a moving frame.

\footnotetext{
${ }^{4}$ An action $G \times M \longrightarrow M,(g, z) \longmapsto g \cdot z$, is called a left action if $g_{1} \cdot\left(g_{2} \cdot z\right)=\left(g_{1} g_{2}\right) \cdot z$ for $g_{1}, g_{2} \in G$ and $z \in M$; or a right action if $g_{1} \cdot\left(g_{2} \cdot z\right)=\left(g_{2} g_{1}\right) \cdot z$, where $g_{1} g_{2}$ and $g_{2} g_{1}$ simply signify the multiplication structure of the group $G$.
} 
Theorem 3.5. Let $M$ be a $G$-space and $G$ act on itself locally freely. Then a moving frame $\rho: M \longrightarrow G$ exists if and only if the action of $G$ on $M$ is locally free.

Proof. If $G$ acts on $M$ locally freely, then we can use the method of choosing a cross-section to the group orbits to construct a moving frame as explained above.

Conversely, suppose that $\rho: M \longrightarrow G$ is a moving frame. Let $z \in M$ be given. There exists an open neighborhood $V$ of the identity element $e$ in $G$ such that, for any $h \in V, \rho(h \cdot z)=h \cdot \rho(z)$. Now, with respect to the action of $G$ on itself, which is a locally free action, the stabilizer of $\rho(z) \in G, G_{\rho(z)}:=\{g \in G \mid g \cdot \rho(z)=\rho(z)\}$, is discrete, and thus there is an open neighborhood $W$ of $e$ in $G$ such that $W \cap G_{\rho(z)}=\{e\}$. Let $G_{z}:=\{h \in G \mid h \cdot z=z\}$ be the stabilizer of $z$. If $h \in(V \cap W) \cap G_{z}$, then $h \cdot \rho(z)=\rho(h \cdot z)=\rho(z)$, implying that $h \in W \cap G_{\rho(z)}$, and thus $h=e$. This means that $G_{z}$ is discrete. Therefore, the action of $G$ on $M$ is locally free.

Remark 3.6. We will adhere to the usual method of using cross-sections to construct moving frames, and $G$ is assumed acting on itself by the rule $g \cdot h \longmapsto h g^{-1}$ as all our examples will involve only left $G$-actions on $M$. Thus when it comes to discussing $G$-invariant differential forms on $G$, such as Maurer-Cartan forms, their invariance shall be meant to be right-invariance unless explicitly specified otherwise.

Now we continue with the previous example and demonstrate how to construct a moving frame.

Example 3.7 (Euclidean plane). Recall from Example 2.3 the prolonged action $\mathcal{A}: \mathrm{SE}(2) \times$ $J^{1}\left(\mathbb{R}, \mathbb{R}^{2}\right) \longrightarrow J^{1}\left(\mathbb{R}, \mathbb{R}^{2}\right)$ of the group $G:=\mathrm{SE}(2)$ on $M:=J^{1}\left(\mathbb{R}, \mathbb{R}^{2}\right)$ in local coordinates: $(\theta, a, b) \cdot\left(x, u, v, u_{x}, v_{x}\right)=\left(x, \widehat{u}, \widehat{v}, \widehat{u_{x}}, \widehat{v_{x}}\right)$. We choose the cross-section to the $\operatorname{SE}(2)$-orbits in $J^{1}\left(\mathbb{R}, \mathbb{R}^{2}\right)$, characterized by the (normalization) equations:

$$
\mathcal{A}^{*} u=\widehat{u}=0, \quad \mathcal{A}^{*} v=\widehat{v}=0, \quad \mathcal{A}^{*} u_{x}=\widehat{u_{x}}=0 .
$$

These equations are solved to find the group parameters

$$
\theta=\tan ^{-1}\left(\frac{u_{x}}{v_{x}}\right), \quad a=\frac{v u_{x}-u v_{x}}{\sqrt{u_{x}^{2}+v_{x}^{2}}}, \quad b=\frac{-u u_{x}-v v_{x}}{\sqrt{u_{x}^{2}+v_{x}^{2}}},
$$

that uniquely determine a group element in a sufficiently small neighborhood of the identity element of $\mathrm{SE}(2)$. Then the corresponding moving frame $\rho: J^{1}\left(\mathbb{R}, \mathbb{R}^{2}\right) \longrightarrow \mathrm{SE}(2)$ is given by

$$
\rho\left(x, u, v, u_{x}, v_{x}\right)=\left(\tan ^{-1}\left(\frac{u_{x}}{v_{x}}\right), \frac{v u_{x}-u v_{x}}{\sqrt{u_{x}^{2}+v_{x}^{2}}}, \frac{-u u_{x}-v v_{x}}{\sqrt{u_{x}^{2}+v_{x}^{2}}}\right)
$$

and its moving frame section $\sigma: J^{1}\left(\mathbb{R}, \mathbb{R}^{2}\right) \longrightarrow \mathrm{SE}(2) \times J^{1}\left(\mathbb{R}, \mathbb{R}^{2}\right)$ is

$$
\sigma\left(x, u, v, u_{x}, v_{x}\right)=\left(\rho\left(x, u, v, u_{x}, v_{x}\right), x, u, v, u_{x}, v_{x}\right) .
$$

The existence of a moving frame implies that $\mathrm{SE}(2)$ acts on $J^{1}\left(\mathbb{R}, \mathbb{R}^{2}\right)$ locally freely according to Theorem 3.5.

In general, let us denote the action of $G$ on $M$ by $\mathcal{A}: G \times M \longrightarrow M,(g, z) \longmapsto g \cdot z:=\mathcal{A}(g, z)$, and define an action of $G$ on $G \times M$ by

$$
G \times(G \times M) \longrightarrow G \times M, \quad(g,(h, z)) \longmapsto(g \cdot h, g \cdot z),
$$

where $g \cdot h$ reflects any locally free action of $G$ on itself such as $g \cdot h \longmapsto h g^{-1}$. Suppose that $\rho: M \longrightarrow G$ is a moving frame, $\sigma: M \longrightarrow G \times M$ its associated moving frame section, and $\pi: G \times M \longrightarrow M$ the canonical projection. 
Proposition 3.8. The maps $\sigma$ and $\pi$ are $G$-equivariant, and $\mathcal{A}$ is $G$-invariant. Also the map $\iota:=\mathcal{A} \circ \sigma: M \longrightarrow M$ is $G$-invariant.

Proof. We will show that the moving frame section $\sigma: M \longrightarrow G \times M$ is equivariant. The other claims about $\pi, \mathcal{A}$, and $\iota$ can all be proved in a similar fashion. Thus suppose that $g \in G$ and $z \in M$. Then $\sigma(g \cdot z)=(\rho(g \cdot z), g \cdot z)=(g \cdot \rho(z), g \cdot z)=g \cdot(\rho(z), z)=g \cdot \sigma(z)$. Therefore $\sigma$ is $G$-equivariant.

All these maps are put together in the following diagram for reference.

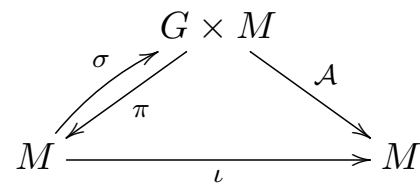

The invariantization operator $\iota^{*}: \Omega^{\bullet}(M) \longrightarrow \Omega^{\bullet}(M)$, where $\Omega^{\bullet}(M):=\bigoplus_{k=0}^{\operatorname{dim} M} \Omega^{k}(M)$ denotes the exterior algebra of differential forms on $M$, turns, thanks to Proposition 3.3, any differential form on $M$ into an invariant differential form, and thus occupies a central position in many works in the literature on the theory and applications of equivariant moving frames.

Example 3.9 (Euclidean plane). The $\mathrm{SE}(2)$-action defined in Example 2.3 on $J^{1}\left(\mathbb{R}, \mathbb{R}^{2}\right)$,

$$
\mathcal{A}: \operatorname{SE}(2) \times J^{1}\left(\mathbb{R}, \mathbb{R}^{2}\right) \longrightarrow J^{1}\left(\mathbb{R}, \mathbb{R}^{2}\right),
$$

pulls back the coordinate functions $\left(x, u, v, u_{x}, v_{x}\right)$ of $J^{1}\left(\mathbb{R}, \mathbb{R}^{2}\right)$ to $\mathrm{SE}(2)$-invariant functions

$$
\begin{aligned}
& \mathcal{A}^{*} x=x, \quad \mathcal{A}^{*} u=u \cos \theta-v \sin \theta+a, \quad \mathcal{A}^{*} v=u \sin \theta+v \cos \theta+b, \\
& \mathcal{A}^{*} u_{x}=u_{x} \cos \theta-v_{x} \sin \theta, \quad \mathcal{A}^{*} v_{x}=u_{x} \sin \theta+v_{x} \cos \theta
\end{aligned}
$$

on $\operatorname{SE}(2) \times J^{1}\left(\mathbb{R}, \mathbb{R}^{2}\right)$. Pulling these functions further back by the moving frame section $\sigma$ : $J^{1}\left(\mathbb{R}, \mathbb{R}^{2}\right) \longrightarrow \mathrm{SE}(2) \times J^{1}\left(\mathbb{R}, \mathbb{R}^{2}\right)$ results in $\mathrm{SE}(2)$-invariant functions on $J^{1}\left(\mathbb{R}, \mathbb{R}^{2}\right)$ :

$$
\begin{aligned}
& \sigma^{*} \mathcal{A}^{*} x=\iota^{*} x=x, \quad \sigma^{*} \mathcal{A}^{*} u=\iota^{*} u=0, \quad \sigma^{*} \mathcal{A}^{*} v=\iota^{*} v=0, \\
& \sigma^{*} \mathcal{A}^{*} u_{x}=\iota^{*} u_{x}=0, \quad \sigma^{*} \mathcal{A}^{*} v_{x}=\iota^{*} v_{x}=\sqrt{u_{x}^{2}+v_{x}^{2}} .
\end{aligned}
$$

In connection with certain coframe fields that we will construct later on (Theorem 4.7), we make the observation that the invariantization of the coordinate functions $u, v, u_{x}$ that were used in setting up the normalization equations (3), necessarily and trivially, yields constants (same as the ones put on the right-hand sides of the normalization equations).

\section{Congruence of immersions}

In this section, we give an answer to our key question: what are the conditions that immersions must satisfy in order for them to be congruent under the action of a Lie group on their ambient space?

First of all, the very definition of prolongation of group actions implies that one can replace a given congruence problem of immersions by a prolonged congruence problem without changing final solutions. More specifically,

Lemma 4.1. Two immersions $\psi, \phi: X \longrightarrow M$ are congruent at $x_{0} \in X$ if and only if, for each $k=0,1, \ldots$, their prolonged graphs $j^{k} \gamma(\psi), j^{k} \gamma(\phi): X \longrightarrow J^{k}(X, M)$ are locally congruent at $x_{0}$ where $G$ acts on $J^{k}(X, M)$ by verticalized prolongation. 
Suppose that $\left\{\omega^{1}, \omega^{2}, \ldots, \omega^{n}\right\}$ is a coframe field on $M$. The coefficients $f_{j k}^{i}$ in their structure equations

$$
\mathrm{d} \omega^{i}=-\frac{1}{2} \sum_{j, k=1}^{n} f_{j k}^{i} \omega^{j} \wedge \omega^{k}, \quad i=1,2, \ldots, n,
$$

where $f_{j k}^{i}+f_{k j}^{i}=0, j, k=1,2, \ldots, n$, are, in general, non-constant functions on $M$. They do become constants if, for example, the coframe field is the Maurer-Cartan coframe field on a Lie group or its generalization on a homogeneous space. Our solution to the congruence problem will rely on a particular kind of invariant coframe fields that have constant structure functions.

Definition 4.2. A coframe field on $M$ is said to be of constant structure if all its structure functions are constant functions.

The following theorem, one of our main results of the paper, finds a necessary and sufficient condition for immersions to be congruent when there exists on the ambient $G$-space a $G$-invariant coframe field of constant structure.

Theorem 4.3. Suppose that an n-dimensional $G$-space $M$ has a $G$-invariant coframe field $\left\{\omega^{i}\right\}$ of constant structure. Let $\psi, \phi: X \longrightarrow M$ be immersions. Then $\psi$ and $\phi$ are congruent at $x_{0} \in X$ if and only if there exist $g \in G$ and $x_{0} \in X$ such that $\psi\left(x_{0}\right)=g \circ \phi\left(x_{0}\right)$ and $\psi^{*} \omega^{i}=\phi^{*} \omega^{i}$, $i=1,2, \ldots, n$, on a neighborhood of $x_{0}$.

Proof. The proof for the "only if" direction is trivial. To prove the "if" direction, let $\pi_{X}$ and $\pi_{M}$ denote the canonical projections of $X \times M$ onto the factors $X$ and $M$, respectively. Suppose that $\left\{C_{j k}^{i}\right\}$ are the structure constants of the coframe field $\left\{\omega^{i}\right\}$ so that for each $i=1,2, \ldots, n$,

$$
\mathrm{d} \omega^{i}=-\frac{1}{2} \sum_{j, k=1}^{n} C_{j k}^{i} \omega^{j} \wedge \omega^{k}
$$

where $C_{j k}^{i}+C_{k j}^{i}=0, j, k=1,2, \ldots, n$. The maps that we are looking at are shown in the following diagram

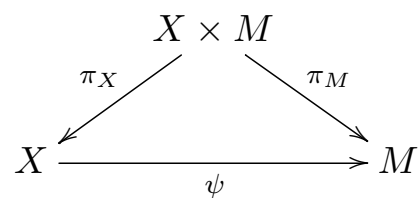

Consider the ideal $\mathcal{I}:=\left\langle\pi_{M}^{*} \omega^{i}-\pi_{X}^{*} \psi^{*} \omega^{i}\right\rangle \subset \Omega^{\bullet}(X \times M)$ generated algebraically by the one-forms $\left\{\pi_{M}^{*} \omega^{i}-\pi_{X}^{*} \psi^{*} \omega^{i}\right\}$. Then, for each $i=1,2, \ldots, n$,

$$
\begin{aligned}
\mathrm{d}\left(\pi_{M}^{*} \omega^{i}-\pi_{X}^{*} \psi^{*} \omega^{i}\right)= & -\frac{1}{2} \sum_{j, k=1}^{n} C_{j k}^{i} \pi_{M}^{*} \omega^{j} \wedge \pi_{M}^{*} \omega^{k}+\frac{1}{2} \sum_{j, k=1}^{n} C_{j k}^{i} \pi_{X}^{*} \psi^{*} \omega^{j} \wedge \pi_{X}^{*} \psi^{*} \omega^{k} \\
= & -\frac{1}{2} \sum_{j, k=1}^{n} C_{j k}^{i} \pi_{M}^{*} \omega^{j} \wedge \pi_{M}^{*} \omega^{k}+\frac{1}{2} \sum_{j, k=1}^{n} C_{j k}^{i} \pi_{M}^{*} \omega^{j} \wedge \pi_{X}^{*} \psi^{*} \omega^{k} \\
& -\frac{1}{2} \sum_{j, k=1}^{n} C_{j k}^{i} \pi_{M}^{*} \omega^{j} \wedge \pi_{X}^{*} \psi^{*} \omega^{k}+\frac{1}{2} \sum_{j, k=1}^{n} C_{j k}^{i} \pi_{X}^{*} \psi^{*} \omega^{j} \wedge \pi_{X}^{*} \psi^{*} \omega^{k} \\
= & -\frac{1}{2} \sum_{j, k=1}^{n} C_{j k}^{i} \pi_{M}^{*} \omega^{j} \wedge\left(\pi_{M}^{*} \omega^{k}-\pi_{X}^{*} \psi^{*} \omega^{k}\right)
\end{aligned}
$$




$$
-\frac{1}{2} \sum_{j, k=1}^{n} C_{j k}^{i}\left(\pi_{M}^{*} \omega^{j}-\pi_{X}^{*} \psi^{*} \omega^{j}\right) \wedge \pi_{X}^{*} \psi^{*} \omega^{k} \equiv 0 \quad \bmod \mathcal{I} .
$$

This shows that $\mathcal{I}$ is a differential ideal. Since, by assumption, $g \circ \phi\left(x_{0}\right)=\psi\left(x_{0}\right)$ for some $g \in G$ and $x_{0} \in X$, and since $(g \circ \phi)^{*} \omega^{i}=\phi^{*} g^{*} \omega^{i}=\phi^{*} \omega^{i}=\psi^{*} \omega^{i}$, the uniqueness result in Cartan's technique of the graph, or Theorem 2.34 in [16], implies that $g \circ \phi(x)=\psi(x)$ for all $x$ in an open neighborhood of $x_{0}$. This completes the proof.

Therefore this theorem essentially turns the congruence problem of immersions into the problem of constructing an invariant coframe field of constant structure. In general, of course, there is no guarantee that a given $G$-space $M$ will admit any invariant coframe field, let alone one of constant structure. However, in many situations, a prolonged verticalized action of $G$ on a jet bundle $J^{k}(X, M)$ may become locally free for some sufficiently large order $k$, which will then lead to the construction of a $k^{\text {th }}$ order moving frame and hence an invariant coframe field on the jet bundle (to be explained below). Once we have in particular a constant-structure invariant coframe field constructed on $J^{k}(X, M)$, we can solve, thanks to Lemma 4.1, the original congruence problem of immersions $\psi: X \longrightarrow M$ by solving, instead, the congruence problem of the prolonged graphs $j^{k} \gamma(\psi): X \longrightarrow J^{k}(X, M)$ of the immersions $\psi$. The following diagram depicts how the immersion $\psi$ in the congruence problem is converted to a prolonged immersion $j^{k} \gamma(\psi)$ of its graph $\gamma(\psi)$ so that we can try our method of using a constant-structure invariant coframe field on $J^{k}(X, M)$

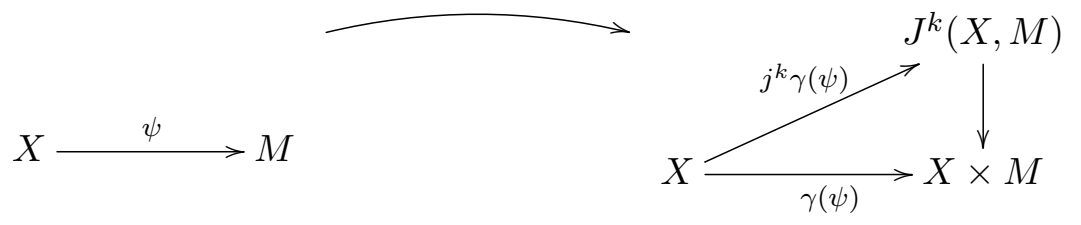

Remark 4.4. We offer a brief perspective, for the reader familiar with the inceptive paper [5], on how our result compares to some part of that work. Griffiths in [5] bases his foundational lemmas determining congruence, proved in the paper's first section, upon the crucial transitivity assumption that any two smooth maps $f$ and $\tilde{f}$ into a Lie group $G$ (or a homogeneous $G$-space) are related by a $G$-valued function $h$ so that $f(x)=h(x) \tilde{f}(x)$ for all $x$ in the domain of the maps. This transitivity assumption is conspicuously absent in our take on the problem, and thus Griffiths' result is subsumed as a special case of our more general result, Theorem 4.3. On the other hand, our constant-structure invariant coframe fields can be interpreted as a natural extension to arbitrary $G$-spaces, homogeneous or not, of Maurer-Cartan coframes (which are always of constant structure) that Griffiths uses to gauge the difference $h$ between the two maps $f$ and $\tilde{f}$.

While Theorem 4.3 does not assume transitivity of the action of $G$ on $M$, and thus can solve the congruence problem of immersions in both homogeneous and nonhomogeneous spaces within one coherent framework, its method requires that the invariant coframe field be of constant structure. Therefore, we proceed to discuss the existence and construction of such a coframe field. We should note that the basic ideas in the following lemmas have originated from the references $[2,3]$.

Let $\pi_{G}: G \times M \longrightarrow G$ denote the canonical projection $(g, z) \longmapsto g$. Let $\left(u^{1}, \ldots, u^{n}\right)$ be the local coordinate system of $M$, and $\left\{\mu^{j} \in \Omega^{1}(G) \mid j=1, \ldots, r\right\}$ the Maurer-Cartan coframe field of $G$.

Lemma 4.5. The pulled-back one-forms

$$
\left\{\mathcal{A}^{*} \mathrm{~d} u^{i}, \pi_{G}^{*} \mu^{j} \in \Omega^{1}(G \times M) \mid i=1, \ldots, n ; j=1, \ldots, r\right\}
$$

form a $G$-invariant coframe field of $G \times M$. 
Proof. The map $\mathcal{A}$ is $G$-invariant and $\pi_{G}$ is $G$-equivariant. (Recall Proposition 3.8.) Thus, by Proposition 3.3, the one-forms (4) are $G$-invariant.

To prove that (4) is a coframe field of $G \times M$, we first note that $\mathcal{A}$ is a submersion since, for any $z \in M$ and $v \in T_{z} M$, we can take $w:=(0, v) \in T_{e} G \oplus T_{z} M \cong T_{(e, z)}(G \times M)$ so that $\mathrm{d} \mathcal{A}(w)=v$. Thus, $\left\{\mathcal{A}^{*} \mathrm{~d} u^{i} \mid i=1, \ldots, n\right\}$ are linearly independent at every point on $G \times M$. Likewise, $\left\{\pi_{G}^{*} \mu^{j} \mid j=1, \ldots, r\right\}$ are linearly independent on $G \times M$ since $\pi_{G}$ is a submersion as well. To show that the union $\left\{\mathcal{A}^{*} \mathrm{~d} u^{i}, \pi_{G}^{*} \mu^{j} \mid i=1, \ldots, n ; j=1, \ldots, r\right\}$ is also linearly independent, it suffices to show that, for any $\xi \in T^{*} G$, the equation $\pi_{G}^{*} \xi=\sum_{i=1}^{n} c_{i} \mathcal{A}^{*} \mathrm{~d} u^{i}, c_{i} \in \mathbb{R}$, forces $c_{i}=0$ for all $i=1, \ldots, n$. Indeed, for any given $z \in M$, take any $w=(0, v) \in T_{(e, z)}(G \times M)$ so that $\mathrm{d} \pi_{G}(w)=0$. Then the equation $\left(\pi_{G}^{*} \xi\right)(w)=\left(\sum_{i=1}^{n} c_{i} \mathcal{A}^{*} \mathrm{~d} u^{i}\right)(w)$ becomes $0=\xi\left(\mathrm{d} \pi_{G}(w)\right)=$ $\sum_{i=1}^{n} c_{i} \mathrm{~d} u^{i}(\mathrm{~d} \mathcal{A}(w))=\sum_{i=1}^{n} c_{i} \mathrm{~d} u^{i}(v)$. Since this equation holds for all $v \in T_{z} M$ and all $z \in M$, it reduces to $0=\sum_{i=1}^{n} c_{i} \mathrm{~d} u^{i}$ on $M$. Thus $c_{i}=0$ for all $i=1, \ldots, n$. Therefore $\left\{\mathcal{A}^{*} \mathrm{~d} u^{i}, \pi_{G}^{*} \mu^{j}\right\}$ are linearly independent at every point of $G \times M$. Finally, since $\operatorname{dim}(G \times M)=r+n$, the set $\left\{\mathcal{A}^{*} \mathrm{~d} u^{i}, \pi_{G}^{*} \mu^{j} \mid i=1, \ldots, n ; j=1, \ldots, r\right\}$ must be a coframe field of $G \times M$.

In the following is shown the prime role in our paper of moving frames and their associated sections, which is to construct constant-structure invariant coframe fields on $M$

Lemma 4.6. If the $G$-action on $M$ is locally free, then there exists on $M$ an invariant coframe field of constant structure.

Proof. Suppose that $\sigma: M \longrightarrow G \times M$ is a moving frame section whose existence is guaranteed by Theorem 3.5. We pull back the $G$-invariant coframe field (4) of $G \times M$ by $\sigma$ to $M$ to obtain

$$
\sigma^{*} \mathcal{A}^{*} \mathrm{~d} u^{i}, \quad i=1, \ldots, n, \quad \sigma^{*} \pi_{G}^{*} \mu^{j}, \quad j=1, \ldots, r .
$$

First, note that, since $\sigma$ is an embedding, the one-forms (5), when evaluated at any point $z \in M$, should span the cotangent space $T_{z}^{*} M$. Thus $n=\operatorname{dim} M$ of the $n+r$ one-forms (5) will form a (local) coframe field of $M$. Also, $G$-invariance of (5) follows from the fact that the one-forms are the pull-backs of the invariant one-forms (4) by the $G$-equivariant map $\sigma$. Finally, the invariant coframe field is of constant structure since $\mathrm{d}\left(\sigma^{*} \mathcal{A}^{*} \mathrm{~d} u^{i}\right)=\mathrm{dd} \sigma^{*} \mathcal{A}^{*} u^{i}=0, i=1, \ldots, n$, and $\mathrm{d}\left(\sigma^{*} \pi_{G}^{*} \mu^{j}\right)=\sigma^{*} \pi_{G}^{*} \mathrm{~d} \mu^{j}, j=1, \ldots, r$, where the Maurer-Cartan coframe field $\left\{\mu^{j}\right\}$ of $G$ is of constant structure.

Recall that, for our usual initial step in constructing a moving frame, we specify a crosssection $\Gamma$ to the $G$-orbits in $M$ by setting up $r=\operatorname{dim} G$ normalization equations $\mathcal{A}^{*} u^{i_{\kappa}}=c_{\kappa}$, $\kappa=1, \ldots, r$, where we typically choose to use constants for $c_{\kappa}$. An implication of the constants $c_{\kappa}$ is that $r$ of the one-forms (5) will necessarily vanish for $\sigma^{*} \mathcal{A}^{*} \mathrm{~d} u^{i_{\kappa}}=\mathrm{d} c_{\kappa}=0$. Therefore this typical approach gives rise to the following final formula for constructing a constant-structure invariant coframe field of $M$. We continue to assume that $G$ acts on $M$ locally freely and that $u^{i}$, $i=1, \ldots, n$, are the local coordinate functions of $M$.

Theorem 4.7. Suppose that, in the course of constructing a moving frame, the $r$ coordinate functions $u^{i}, i=1, \ldots, r$, of $M$ are normalized to constants; that is, $\iota^{*} u^{i}=\sigma^{*} \mathcal{A}^{*} u^{i}=c_{i}$, constant. Then $M$ has the following constant-structure $G$-invariant coframe field:

$$
\sigma^{*} \mathcal{A}^{*} \mathrm{~d} u^{i}=\mathrm{d} \sigma^{*} \mathcal{A}^{*} u^{i}=\mathrm{d} \iota^{*} u^{i}, \quad i=r+1, \ldots, n, \quad \sigma^{*} \pi_{G}^{*} \mu^{j}=\rho^{*} \mu^{j}, \quad j=1, \ldots, r .
$$

Remark 4.8. Note that, if a coordinate function $u^{\kappa}$ of $M$ is normalized to a constant $c_{\kappa}$ in the process of choosing a cross-section $\Gamma$ to the group orbits, then $\mathrm{d} \iota^{*} u^{\kappa}=\mathrm{d} c_{\kappa}=0$. Thus, even if 
we implement the formula (5) for every coordinate function $u^{i}, i=1, \ldots, n$, it will not cause any problem in constructing our desired constant-structure invariant coframe field, apart from the potential unexciting prospect of having to spend some extra time computing for those vanishing phantom differential forms.

Now suppose that $\left(x^{1}, x^{2}, \ldots, x^{p}\right)$ are local coordinate functions on $X$ and that a constantstructure invariant coframe field $\left\{\omega^{i}=\sum_{j=1}^{n} f_{j}^{i} \mathrm{~d} u^{j} \mid i=1,2, \ldots, n\right\}$ exists on $M$ with some functions $f_{j}^{i}: M \longrightarrow \mathbb{R}$. Then, assuming $p=\operatorname{dim} X$, the pull-backs

$$
\psi^{*} \omega^{i}=\sum_{l=1}^{p} \sum_{j=1}^{n}\left(f_{j}^{i} \circ \psi\right) \frac{\partial\left(u^{j} \circ \psi\right)}{\partial x^{l}} \mathrm{~d} x^{l}, \quad i=1,2, \ldots, n,
$$

by congruent immersions $\psi: X \longrightarrow M$ must all agree by Theorem 4.3 . In turn, these pulledback forms will agree if and only if the differential functions $\left\{\sum_{j=1}^{n} f_{j}^{i} u_{l}^{j}\right\}$, where $u_{l}^{j}$ are the local coordinate functions on the fibers of the bundle $J^{1}(X, M) \longrightarrow X \times M$, agree when pulled back by the immersions $\psi$. Thus we have proved:

Lemma 4.9. The differential functions $\left\{\sum_{j=1}^{n} f_{j}^{i} u_{l}^{j} \mid i=1, \ldots, n ; l=1, \ldots, p\right\}$ form a complete system of congruence invariants for immersions from $X$ to $M$.

Remark 4.10. In general, the complete system $\left\{\sum_{j=1}^{n} f_{j}^{i} u_{l}^{j}\right\}$ will not be functionally independent, and thus there will be some functional redundancy in the system, as is indeed the case with most of the examples in our paper. Also, a subset consisting of only functionally independent invariants may still be further reduced to an even smaller subset consisting of lower order differential invariants by employing the idea of invariant differential operators, [11], that can be used to eliminate differential redundancy. Related to this point, it should be noted that the case of variable-parameter equivalence problems has in recent years witnessed major advances made by Olver et al. both in the general approach to obtaining (minimal) generating systems of differential invariants and in its applications to some specific geometric settings; see $[6,10,12,13]$. In our present paper, we will not venture into this intricate question of minimalizing systems of invariants; the interested reader should instead consult the cited references.

In general, a group action on $M$ may not be locally free, and so a constant-structure invariant coframe field may not be available on $M$. However, if we prolong the verticalized action on $X \times M$ of the group to a jet bundle $J^{k}(X, M)$ of a sufficiently high order $k$, the resulting action may become locally free essentially thanks to, intuitively speaking, the wider space that mitigates the crowding of the group orbits. Once we have a locally free action on a jet bundle $J^{k}(X, M)$, we can apply the ideas of Theorems 4.3 and 4.7 to construct a constant-structure invariant coframe field on $J^{k}(X, M)$ and solve the congruence problem.

The following result tells us basically how many invariants at most, in terms of their differential order, we should require to resolve a congruence problem.

Corollary 4.11. Suppose that a $k^{\text {th }}$ order jet bundle $J^{k}(X, M)$ admits a constant-structure $G$-invariant coframe field. Then the differential order of a minimal complete set of congruence invariants is bounded by $k+1$.

Proof. Suppose that $\left\{\sum_{l=1}^{p} h_{l}^{\alpha} \mathrm{d} x^{l}, \sum_{|J|=0}^{k} \sum_{j=1}^{n} f_{i, j}^{I, J} \mathrm{~d} u_{J}^{j}\right\}$ (where $\alpha=1, \ldots, p, i=1, \ldots, n$, and $I$ is a symmetric multi-index over $\{1, \ldots, p\}$ of order up to $k$ ) is a constant-structure invariant 
coframe field on $J^{k}(X, M)$. Note that all the differential functions $h_{l}^{\alpha}, x^{l}, f_{i, j}^{I, J}$, and $u_{J}^{j}$ are of order up to $k$. Then, by Lemma 4.9 and with the understanding that $M$ and $\psi$ in the proof of the lemma are now replaced respectively by $J^{k}(X, M)$ and $j^{k} \gamma(\psi)$, a minimal complete set of congruence invariants must be a subset of $\left\{h_{l}^{\alpha}, \sum_{|J|=0}^{k} \sum_{j=1}^{n} f_{i, j}^{I, J} u_{J, l}^{j}\right\}$ which is of differential order $k+1$ or less.

Thus, for example, if the verticalized prolongation of the group action becomes locally free at order $k$, then the conclusion of the corollary holds true.

Remark 4.12. Note that fixed-parameter congruence is a stronger condition than variableparameter congruence. Thus, Corollary 4.11 implies in the case of variable-parameter congruence problems that, under the assumptions of the corollary, the differential order of a minimal complete set of variable-parameter congruence invariants is also bounded by $k+1$.

Once a constant-structure invariant coframe field $\left\{\omega^{i}\right\}$ exists, any other invariant coframe field $\left\{\zeta^{i}\right\}$ of the form

$$
\zeta^{i}:=\sum_{j=1}^{n} a_{j}^{i} \omega^{j}, \quad a_{j}^{i}=\text { const }, \quad i=1,2, \ldots, n,
$$

will be of constant structure, and can also be used for solving the congruence problem of immersions. If, however, an invariant coframe field $\left\{\zeta^{i}\right\}$ is not of constant structure, then at least necessary conditions for congruence can be obtained.

Proposition 4.13. Suppose that $M$ admits an invariant coframe field of constant structure and that $\left\{\zeta^{i}\right\}$ is any invariant coframe field that may not be of constant structure. If two immersions $\psi, \phi: X \longrightarrow M$ are congruent, then $\psi^{*} \zeta^{i}=\phi^{*} \zeta^{i}$ for all $i=1,2, \ldots, n$.

Proof. Let $\left\{\omega^{i}\right\}$ be a constant-structure invariant coframe field on $M$. Suppose $\psi=g \circ \phi$ for some $g \in G$. Since $\left\{\zeta^{i}\right\}$ is an invariant coframe field, there exist invariant functions $f_{j}^{i}$ on $M$ such that $\zeta^{i}=\sum_{j=1}^{n} f_{j}^{i} \omega^{j}$ for all $i=1, \ldots, n$. Then for each $i$,

$$
\psi^{*} \zeta^{i}=\sum_{j=1}^{n}\left((g \circ \phi)^{*} f_{j}^{i}\right)\left((g \circ \phi)^{*} \omega^{j}\right)=\sum_{j=1}^{n}\left(\phi^{*} g^{*} f_{j}^{i}\right)\left(\phi^{*} g^{*} \omega^{j}\right)=\sum_{j=1}^{n}\left(\phi^{*} f_{j}^{i}\right)\left(\phi^{*} \omega^{j}\right)=\phi^{*} \zeta^{i} .
$$

Remark 4.14. Fortunately, we have the general formula (6) for constructing constant-structure invariant coframe fields that will enable us to solve congruence problems completely and obtain necessary and sufficient conditions for congruence.

Example 4.15 (Euclidean plane). We continue the discussion and notation of the SE(2)-action that we had in the earlier examples. To find a complete system of $\mathrm{SE}(2)$-congruence invariants of curve immersions in Euclidean plane, we need to construct a constant-structure SE(2)-invariant coframe field on $J^{1}\left(\mathbb{R}, \mathbb{R}^{2}\right)$ where we confirmed that $\mathrm{SE}(2)$ acts locally freely. We follow the formula (6) to construct such a coframe field. According to the formula, part of the coframe field comes from the Maurer-Cartan coframe field of $\mathrm{SE}(2)$, which we find by first embedding $\mathrm{SE}(2)$ in $\mathrm{GL}(3)$ :

$$
\mathrm{SE}(2) \longrightarrow \mathrm{GL}(3, \mathbb{R}), \quad(\theta, a, b) \longmapsto g:=\left[\begin{array}{ccc}
\cos \theta & -\sin \theta & a \\
\sin \theta & \cos \theta & b \\
0 & 0 & 1
\end{array}\right],
$$


which acts on the hyperplane $\left\{\left[\begin{array}{lll}u & v & 1\end{array}\right]^{\mathrm{T}} \in \mathbb{R}^{3} \mid u, v \in \mathbb{R}\right\}$ via matrix multiplication, and then taking the linearly independent entries of the $\mathfrak{g l}(3, \mathbb{R})$-valued Maurer-Cartan form $(\mathrm{d} g) g^{-1}$ :

$$
\mu^{1}:=\mathrm{d} \theta, \quad \mu^{2}:=\mathrm{d} a+b \mathrm{~d} \theta, \quad \mu^{3}:=\mathrm{d} b-a \mathrm{~d} \theta .
$$

We pull these Maurer-Cartan forms by the moving frame $\rho: J^{1}\left(\mathbb{R}, \mathbb{R}^{2}\right) \longrightarrow \mathrm{SE}(2)$ to obtain the invariant one-forms on $J^{1}\left(\mathbb{R}, \mathbb{R}^{2}\right)$ :

$$
\rho^{*} \mu^{1}=\frac{v_{x} \mathrm{~d} u_{x}-u_{x} \mathrm{~d} v_{x}}{u_{x}^{2}+v_{x}^{2}}, \quad \rho^{*} \mu^{2}=\frac{-v_{x} \mathrm{~d} u+u_{x} \mathrm{~d} v}{\sqrt{u_{x}^{2}+v_{x}^{2}}}, \quad \rho^{*} \mu^{3}=\frac{-u_{x} \mathrm{~d} u-v_{x} \mathrm{~d} v}{\sqrt{u_{x}^{2}+v_{x}^{2}}}
$$

which will be part of the coframe field that we are trying to construct.

The other part of the formula (6) is implemented to obtain more invariant one-forms on $J^{1}\left(\mathbb{R}, \mathbb{R}^{2}\right)$ :

$$
\mathrm{d} \iota^{*} x=\mathrm{d} x, \quad \mathrm{~d} \iota^{*} v_{x}=\frac{u_{x}}{\sqrt{u_{x}^{2}+v_{x}^{2}}} \mathrm{~d} u_{x}+\frac{v_{x}}{\sqrt{u_{x}^{2}+v_{x}^{2}}} \mathrm{~d} v_{x},
$$

that complement (7) to form a constant-structure invariant coframe field of $J^{1}\left(\mathbb{R}, \mathbb{R}^{2}\right)$. The final step, suggested by Theorem 4.3, is to pull these coframe forms by the prolonged graph $j^{1} \gamma(\psi)$ of a generic curve immersion $\psi: \mathbb{R} \longrightarrow \mathbb{R}^{2}, x \longmapsto(u(x), v(x))$ :

$$
\begin{aligned}
& \left(j^{1} \gamma(\psi)\right)^{*} \mu^{1}=\frac{u_{x x} v_{x}-u_{x} v_{x x}}{u_{x}^{2}+v_{x}^{2}} \mathrm{~d} x, \quad\left(j^{1} \gamma(\psi)\right)^{*} \mu^{2}=0, \\
& \left(j^{1} \gamma(\psi)\right)^{*} \mu^{3}=-\sqrt{u_{x}^{2}+v_{x}^{2}} \mathrm{~d} x, \quad\left(j^{1} \gamma(\psi)\right)^{*} \mathrm{~d} \iota^{*} x=\mathrm{d} x \\
& \left(j^{1} \gamma(\psi)\right)^{*} \mathrm{~d} \iota^{*} v_{x}=\frac{u_{x} u_{x x}+v_{x} v_{x x}}{\sqrt{u_{x}^{2}+v_{x}^{2}}} \mathrm{~d} x .
\end{aligned}
$$

Therefore, the coefficient functions of $\mathrm{d} x$ in (8),

$$
\frac{u_{x x} v_{x}-u_{x} v_{x x}}{u_{x}^{2}+v_{x}^{2}}, \quad-\sqrt{u_{x}^{2}+v_{x}^{2}}, \quad \frac{u_{x} u_{x x}+v_{x} v_{x x}}{\sqrt{u_{x}^{2}+v_{x}^{2}}},
$$

constitute a complete system of $\mathrm{SE}(2)$-invariants for curve immersions in $\mathbb{R}^{2}$. According to Theorem 4.3, two immersions $\mathbb{R} \longrightarrow \mathbb{R}^{2}$ are congruent if and only if the functions (9), when restricted to the two (prolonged) immersed submanifolds, yield the same values at every point in an open subset of the domain $\mathbb{R}$ of the immersions and also there is a transformation $g \in \operatorname{SE}(2)$ that sends some first-order jet $\left(\widetilde{x}, \widetilde{u}, \widetilde{v}, \widetilde{u_{x}}, \widetilde{v_{x}}\right) \in J^{1}\left(\mathbb{R}, \mathbb{R}^{2}\right)$ of one of the curves to a jet of the other.

Note that the first two congruence invariants $\frac{u_{x x} v_{x}-u_{x} v_{x x}}{u_{x}^{2}+v_{x}^{2}}$ and $-\sqrt{u_{x}^{2}+v_{x}^{2}}$ generate algebraically a space of functions equivalent to the space generated by the well-known curvature and speed invariants of Euclidean planar curves. The third invariant $\frac{u_{x} u_{x x}+v_{x} v_{x x}}{\sqrt{u_{x}^{2}+v_{x}^{2}}}$ is a differential consequence of the second invariant, and hence does not provide any new information.

\section{$5 \quad$ More examples}

\subsection{A homogeneous space: $\mathbb{C P}^{1}$ under the action of $\operatorname{PSL}(2, \mathbb{C})$}

The example of planar curves used in the previous sections was for the congruence problem of immersions in the homogeneous space $\left(\mathbb{R}^{2}, \mathrm{SE}(2)\right)$. In this section, we deal with yet another example of a homogeneous space. 
Consider the action by linear fractional (or Möbius) transformations

$$
\mathrm{SL}(2, \mathbb{C}) \times \mathbb{C} \longrightarrow \mathbb{C}, \quad\left[\begin{array}{ll}
a & b \\
c & d
\end{array}\right] \cdot z \longmapsto \frac{a z+b}{c z+d},
$$

which is a local description of the transitive action

$$
\operatorname{PSL}(2, \mathbb{C}) \times \mathbb{C P}^{1} \longrightarrow \mathbb{C P}^{1}, \quad \overline{\left[\begin{array}{ll}
a & b \\
c & d
\end{array}\right]} \cdot \overline{\left[\begin{array}{c}
z \\
w
\end{array}\right]} \longmapsto \overline{\left[\begin{array}{l}
a z+b w \\
c z+d w
\end{array}\right]},
$$

where the overlines represent the standard projections to the respective quotient spaces $\mathrm{PSL}(2, \mathbb{C})$ $=\mathrm{SL}(2, \mathbb{C}) /\left(\mathrm{SL}(2, \mathbb{C}) \cap \mathbb{C}^{*} I\right)$ and $\mathbb{C P}^{1}=\left(\mathbb{C}^{2}-\{(0,0)\}\right) / \sim$.

We will study local congruence of holomorphic maps $\psi: \mathbb{C P}^{1} \longrightarrow \mathbb{C P}^{1}$ with nonvanishing derivatives. To do so, we first rewrite the action (10) using the parametrization of $\mathrm{SL}(2, \mathbb{C})$ by $(a, b, c)$ :

$$
\mathrm{SL}(2, \mathbb{C}) \times \mathbb{C} \longrightarrow \mathbb{C}, \quad\left[\begin{array}{cc}
a & b \\
c & (b c+1) / a
\end{array}\right] \cdot z \longmapsto \frac{a^{2} z+a b}{a c z+b c+1},
$$

and also view the immersions in local coordinate charts of $\mathbb{C P}^{1}$ so that they are of the form $\psi: \mathbb{C} \longrightarrow \mathbb{C}$. Next, we take the graph of $\psi$,

$$
\gamma(\psi): \mathbb{C} \longrightarrow \mathbb{C} \times \mathbb{C}, \quad z \longmapsto(z, \psi(z)),
$$

where $\operatorname{SL}(2, \mathbb{C})$ acts on $\mathbb{C} \times \mathbb{C}$ by verticalization:

$$
\mathrm{SL}(2, \mathbb{C}) \times(\mathbb{C} \times \mathbb{C}) \longrightarrow \mathbb{C} \times \mathbb{C}, \quad(a, b, c) \cdot(w, z) \longmapsto\left(w, \frac{a^{2} z+a b}{a c z+b c+1}\right)
$$

This action is not locally free, which is obvious from consideration of the dimensions of $\mathrm{PSL}(2, \mathbb{C})$ and $\mathbb{C} \times \mathbb{C}$, and thus we need to try prolonging the action to jet bundles over $\mathbb{C} \times \mathbb{C}$ and see if the resulting action is locally free there. In fact, the prolonged action of $\operatorname{SL}(2, \mathbb{C})$ on $J^{2}(\mathbb{C}, \mathbb{C})$ turns out to be locally free. Specifically, the second-order prolonged action $\mathcal{A}: \operatorname{SL}(2, \mathbb{C}) \times J^{2}(\mathbb{C}, \mathbb{C}) \longrightarrow$ $J^{2}(\mathbb{C}, \mathbb{C})$ is given by

$$
\begin{aligned}
& (a, b, c) \cdot\left(w, z, z_{w}, z_{w w}\right) \\
& \qquad\left(w, \frac{a^{2} z+a b}{a c z+b c+1}, \frac{a^{2} z_{w}}{(a c z+b c+1)^{2}}, \frac{-2 a^{3} c z_{w}^{2}+\left(a^{2}+a^{2} b c\right) z_{w w}+a^{3} c z z_{w w}}{(a c z+b c+1)^{3}}\right)
\end{aligned}
$$

where $\left(w, z, z_{w}, z_{w w}\right)$ denotes the local coordinate system on $J^{2}(\mathbb{C}, \mathbb{C})$. At this point, the chosen (normalization) equations

$$
\begin{aligned}
& \mathcal{A}^{*} z=\frac{a^{2} z+a b}{a c z+b c+1}=0, \quad \mathcal{A}^{*} z_{w}=\frac{a^{2} z_{w}}{(a c z+b c+1)^{2}}=1, \\
& \mathcal{A}^{*} z_{w w}=\frac{-2 a^{3} c z_{w}^{2}+\left(a^{2}+a^{2} b c\right) z_{w w}+a^{3} c z z_{w w}}{(a c z+b c+1)^{3}}=0,
\end{aligned}
$$

can be solved, where $z_{w} \neq 0$, for the group parameters

$$
a=\frac{1}{\sqrt{z_{w}}}, \quad b=-\frac{z}{\sqrt{z_{w}}}, \quad c=\frac{z_{w w}}{2 z_{w} \sqrt{z_{w}}},
$$

which implies that the group action is locally free, and defines the moving frame

$$
\rho: J^{2}(\mathbb{C}, \mathbb{C}) \longrightarrow \mathrm{SL}(2, \mathbb{C}), \quad\left(w, z, z_{w}, z_{w w}\right) \longmapsto(a, b, c),
$$


and the associated section

$$
\sigma: J^{2}(\mathbb{C}, \mathbb{C}) \longrightarrow \mathrm{SL}(2, \mathbb{C}) \times J^{2}(\mathbb{C}, \mathbb{C}), \quad\left(w, z, z_{w}, z_{w w}\right) \longmapsto\left(a, b, c, w, z, z_{w}, z_{w w}\right) .
$$

Now we recall the formula (6) to construct a constant-structure $\operatorname{SL}(2, \mathbb{C})$-invariant coframe field on $J^{2}(\mathbb{C}, \mathbb{C})$. Among the coordinate functions $\left(w, z, z_{w}, z_{w w}\right)$ of $J^{2}(\mathbb{C}, \mathbb{C}), w$ was the one that was not used in the normalization equations (11) and thus it leads to the invariant one-form

$$
\mathrm{d} \iota^{*} w=\mathrm{d} w
$$

on $J^{2}(\mathbb{C}, \mathbb{C})$ as part of the coframe field that we are constructing. The other part of the coframe field requires, according to (6), that we first find Maurer-Cartan forms of $\operatorname{SL}(2, \mathbb{C})$. Thus, assuming

$$
g:=\left[\begin{array}{cc}
a & b \\
c & (b c+1) / a
\end{array}\right] \in \mathrm{SL}(2, \mathbb{C}),
$$

we take the linearly independent entries of the $\mathfrak{s l}(2, \mathbb{C})$-valued Maurer-Cartan form $(\mathrm{d} g) g^{-1}$ to obtain the Maurer-Cartan coframe field of $\operatorname{SL}(2, \mathbb{C})$ :

$$
\mu^{1}:=\frac{b c+1}{a} \mathrm{~d} a-c \mathrm{~d} b, \quad \mu^{2}:=-b \mathrm{~d} a+a \mathrm{~d} b, \quad \mu^{3}:=\frac{b c^{2}+c}{a^{2}} \mathrm{~d} a-\frac{c^{2}}{a} \mathrm{~d} b+\frac{1}{a} \mathrm{~d} c .
$$

Pulling back these Maurer-Cartan forms by the moving frame $\rho: J^{2}(\mathbb{C}, \mathbb{C}) \longrightarrow \operatorname{SL}(2, \mathbb{C})$ yields the following invariant one-forms on $J^{2}(\mathbb{C}, \mathbb{C})$ :

$$
\rho^{*} \mu^{1}=\frac{z_{w w}}{2 z_{w}^{2}} \mathrm{~d} z-\frac{1}{2 z_{w}} \mathrm{~d} z_{w}, \quad \rho^{*} \mu^{2}=-\frac{1}{z_{w}} \mathrm{~d} z, \quad \rho^{*} \mu^{3}=\frac{z_{w w}^{2}}{4 z_{w}^{3}} \mathrm{~d} z-\frac{z_{w w}}{z_{w}^{2}} \mathrm{~d} z_{w}+\frac{1}{2 z_{w}} \mathrm{~d} z_{w w}
$$

that, together with $(12)$, form a constant-structure $\operatorname{SL}(2, \mathbb{C})$-invariant coframe field on $J^{2}(\mathbb{C}, \mathbb{C})$.

Now the final step is to pull back the one-forms in the coframe by the prolonged graph $j^{2} \gamma(\psi)$ of a generic holomorphic immersion $\psi: \mathbb{C} \longrightarrow \mathbb{C}, w \longmapsto z(w)$, to obtain:

$$
\begin{aligned}
& \left(j^{2} \gamma(\psi)\right)^{*} \mathrm{~d} \iota^{*} w=\mathrm{d} w, \quad\left(j^{2} \gamma(\psi)\right)^{*} \rho^{*} \mu^{1}=0, \quad\left(j^{2} \gamma(\psi)\right)^{*} \rho^{*} \mu^{2}=-\mathrm{d} w, \\
& \left(j^{2} \gamma(\psi)\right)^{*} \rho^{*} \mu^{3}=\left(\frac{z_{w w w}}{2 z_{w}}-\frac{3 z_{w w}^{2}}{4 z_{w}^{2}}\right) \mathrm{d} w .
\end{aligned}
$$

Thus $\frac{z_{w w w}}{2 z_{w}}-\frac{3 z_{w w}^{2}}{4 z_{w}^{2}}$ is the only non-constant invariant in this example, and therefore, according to Theorem 4.3, two immersions $\mathbb{C} \longrightarrow \mathbb{C}$ are congruent under linear fractional transformations (10) or two immersions $\mathbb{C P}^{1} \longrightarrow \mathbb{C P}^{1}$ are congruent under the action of $\operatorname{PSL}(2, \mathbb{C})$ if and only if the restriction of the function $\frac{z_{w w w}}{2 z_{w}}-\frac{3 z_{w w}^{2}}{4 z_{w}^{2}}$ on the two (prolonged) immersed submanifolds agree and also there exists an element $g$ of the group that takes some jet $\left(\widetilde{w}, \widetilde{z}, \widetilde{z_{w}}, \widetilde{z_{w w}}\right) \in$ $J^{2}(\mathbb{C}, \mathbb{C})$ of one of the immersions to a jet of the other immersion. The function $\frac{z_{w w w}}{2 z_{w}}-\frac{3 z_{w w}^{2}}{4 z_{w}^{2}}$, therefore, is an $\operatorname{SL}(2, \mathbb{C})$ - or $\operatorname{PSL}(2, \mathbb{C})$-congruence invariant, and, when multiplied by 2 , is in fact known as the Schwarzian derivative.

\subsection{A nonhomogeneous space: $\mathbb{R}^{3}$ under the action of $\mathrm{SO}(3)$}

Consider the action of $\mathrm{SO}(3)$ on $\mathbb{R}^{3}$ via the standard rotations. Note that the resulting space $\left(\mathbb{R}^{3}, \mathrm{SO}(3)\right)$ constitutes a nonhomogeneous space. We will treat the congruence problem of orientable regular hypersurfaces in this space using the same technique that we used for congruence problems for homogeneous spaces. 
For this example, however, we will take a somewhat implicit way of describing the group elements, as opposed to the explicit parametric descriptions of symmetry groups that we used for the other examples. Although it is possible to describe $\mathrm{SO}(3)$ by parameters, such as Euler angles, still arriving at the same final solution to our congruence problem, an implicit description of $\mathrm{SO}(3)$ turns out not only to reduce greatly the amount of computation that would otherwise be required by explicit presence of parameters, but also to give us a better geometric understanding, allowing for deft manipulation, of a moving frame that we will construct.

Thus, any matrix element of the group $\mathrm{SO}(3)$ will from now on be denoted simply by the letter $R$ without referring to any parameters. Also, in order not to clutter expressions with too many different symbols, we will continue the tradition of using same symbols for multiple purposes, supported at times by the customary canonical identification of a vector space such as $\mathbb{R}^{3}$ with its tangent spaces at various points, insofar as there is no danger of serious confusion; in particular, we will use the column matrix notation $u:=\left[u^{1} u^{2} u^{3}\right]^{\mathrm{T}}$ to denote, depending on the context, the coordinate system of $\mathbb{R}^{3}$, a point as a vector in $\mathbb{R}^{3}$, or the image of an immersion $\mathbb{R}^{2} \longrightarrow \mathbb{R}^{3}$.

As always, we view any regular surface $\psi: \mathbb{R}^{2} \longrightarrow \mathbb{R}^{3}$ in terms of its graph

$$
\gamma(\psi): \mathbb{R}^{2} \longrightarrow \mathbb{R}^{2} \times \mathbb{R}^{3}, \quad\left(x^{1}, x^{2}\right) \longrightarrow\left(x^{1}, x^{2}, \psi\left(x^{1}, x^{2}\right)\right),
$$

where $x:=\left(x^{1}, x^{2}\right)$ denotes the coordinate system of the domain $\mathbb{R}^{2}$. We verticalize the action of $\mathrm{SO}(3)$ to the corresponding action on $\mathbb{R}^{2} \times \mathbb{R}^{3}$ :

$$
\mathrm{SO}(3) \times\left(\mathbb{R}^{2} \times \mathbb{R}^{3}\right) \longrightarrow \mathbb{R}^{2} \times \mathbb{R}^{3}, \quad R \cdot(x, u) \longmapsto(x, R u),
$$

where $R u$ signifies matrix multiplication. Since this action is not locally free, we try prolonging the action in an attempt to obtain a locally free action:

$$
\mathcal{A}: \operatorname{SO}(3) \times J^{1}\left(\mathbb{R}^{2}, \mathbb{R}^{3}\right) \longrightarrow J^{1}\left(\mathbb{R}^{2}, \mathbb{R}^{3}\right), \quad R \cdot\left(x, u, u_{1}, u_{2}\right) \longmapsto\left(x, R u, R u_{1}, R u_{2}\right),
$$

where $u_{1}:=\left[\begin{array}{lll}u_{1}^{1} & u_{1}^{2} & u_{1}^{3}\end{array}\right]^{\mathrm{T}}$ and $u_{2}:=\left[\begin{array}{lll}u_{2}^{1} & u_{2}^{2} & u_{2}^{3}\end{array}\right]^{\mathrm{T}}$ denote the fiber coordinates of the bundle $J^{1}\left(\mathbb{R}^{2}, \mathbb{R}^{3}\right) \longrightarrow \mathbb{R}^{2} \times \mathbb{R}^{3}$. Indeed, this first prolongation of the action is locally free since we can solve, for the group element $R$, the following chosen (normalization) equations

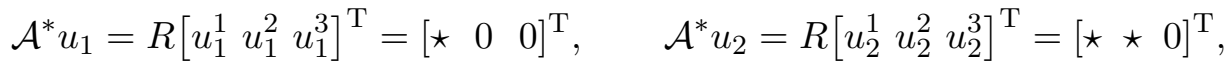

where $\star$ means that the entry is not normalized and hence left free. To help describe the solution $R$ satisfying the equations (13), we need first define a few $\mathbb{R}^{3}$-valued vector fields along surfaces:

$$
\mathbf{n}:=\frac{u_{1} \times u_{2}}{\left\|u_{1} \times u_{2}\right\|}, \quad \mathbf{t}:=\frac{u_{1}}{\left\|u_{1}\right\|}, \quad \mathbf{v}:=\mathbf{n} \times \mathbf{t} .
$$

Note that these ordered vector fields form an oriented orthonormal frame field of $\mathbb{R}^{3}$, restricted to surfaces, possessing the same orientation as the standard one for $\mathbb{R}^{3}$. If $R$ satisfies the equations (13), then

$$
\begin{aligned}
& R \mathbf{n}=\left[\begin{array}{lll}
0 & 0 & 1
\end{array}\right]^{\mathrm{T}}, \quad R \mathbf{t}=\left[\begin{array}{lll}
1 & 0 & 0
\end{array}\right]^{\mathrm{T}}, \quad \text { and } \\
& R \mathbf{v}=R(\mathbf{n} \times \mathbf{t})=(R \mathbf{n}) \times(R \mathbf{t})=\left[\begin{array}{lll}
0 & 0 & 1
\end{array}\right]^{\mathrm{T}} \times\left[\begin{array}{lll}
1 & 0 & 0
\end{array}\right]^{\mathrm{T}}=\left[\begin{array}{lll}
0 & 1 & 0
\end{array}\right]^{\mathrm{T}} .
\end{aligned}
$$

Thus,

$$
R^{-1}\left[\begin{array}{lll}
1 & 0 & 0
\end{array}\right]^{\mathrm{T}}=\mathbf{t}, \quad R^{-1}\left[\begin{array}{lll}
0 & 1 & 0
\end{array}\right]^{\mathrm{T}}=\mathbf{v}, \quad R^{-1}\left[\begin{array}{lll}
0 & 0 & 1
\end{array}\right]^{\mathrm{T}}=\mathbf{n},
$$

and therefore

$$
R=\left[\begin{array}{lll}
\mathbf{t} & \mathbf{v} & \mathbf{n}
\end{array}\right]^{-1}=\left[\begin{array}{lll}
\mathbf{t} & \mathbf{v} & \mathbf{n}
\end{array}\right]^{\mathrm{T}} .
$$


This solution $R$ is what is used in our construction of a moving frame:

$$
\rho: J^{1}\left(\mathbb{R}^{2}, \mathbb{R}^{3}\right) \longrightarrow \mathrm{SO}(3), \quad\left(x, u, u_{1}, u_{2}\right) \longmapsto[\mathbf{t} \mathbf{v} \mathbf{n}]^{\mathrm{T}},
$$

and its associated section

$$
\sigma: J^{1}\left(\mathbb{R}^{2}, \mathbb{R}^{3}\right) \longrightarrow \mathrm{SO}(3) \times J^{1}\left(\mathbb{R}^{2}, \mathbb{R}^{3}\right), \quad\left(x, u, u_{1}, u_{2}\right) \longmapsto\left([\mathbf{t} \mathbf{v} \mathbf{~ n}]^{\mathrm{T}}, x, u, u_{1}, u_{2}\right) .
$$

To construct a constant-structure $\mathrm{SO}(3)$-invariant coframe field on $J^{1}\left(\mathbb{R}^{2}, \mathbb{R}^{3}\right)$, we now implement the formula (6) while having in mind Remark 4.8 and the definition $\iota:=\mathcal{A} \circ \sigma$,

$$
\begin{aligned}
& \mathrm{d} \iota^{*} x=\mathrm{d} x, \\
& \mathrm{~d} \iota^{*} u=\mathrm{d} \sigma^{*}(R u)=\mathrm{d}\left(\left(\rho^{*} R\right) u\right)=\mathrm{d}\left(\left[\begin{array}{ll}
\mathbf{t} & \mathbf{n}
\end{array}\right]^{\mathrm{T}} u\right)=[\mathrm{d} \mathbf{t} \mathrm{d} \mathbf{v} \mathrm{d} \mathbf{n}]^{\mathrm{T}} u+\left[\begin{array}{ll}
\mathbf{t} & \mathbf{n}
\end{array}\right]^{\mathrm{T}} \mathrm{d} u, \\
& \mathrm{~d} \iota^{*} u_{1}=\left[\begin{array}{lll}
\mathrm{d} \mathbf{t} & \mathrm{d} \mathbf{v} & \mathrm{d} \mathbf{n}
\end{array}\right]^{\mathrm{T}} u_{1}+\left[\begin{array}{lll}
\mathbf{t} & \mathbf{v} & \mathbf{n}
\end{array}\right]^{\mathrm{T}} \mathrm{d} u_{1}, \\
& \mathrm{~d} \iota^{*} u_{2}=\left[\begin{array}{lll}
\mathrm{d} \mathbf{t} & \mathrm{d} \mathbf{v} & \mathrm{d} \mathbf{n}
\end{array}\right]^{\mathrm{T}} u_{2}+\left[\begin{array}{lll}
\mathbf{t} & \mathbf{v} & \mathbf{n}
\end{array}\right]^{\mathrm{T}} \mathrm{d} u_{2} .
\end{aligned}
$$

We also pull back, by the moving frame $\rho$, the $\mathfrak{s o}(3)$-valued Maurer-Cartan form $(\mathrm{d} R) R^{-1}$ of $\mathrm{SO}(3)$ to $J^{1}\left(\mathbb{R}^{2}, \mathbb{R}^{3}\right)$ :

$$
\rho^{*}\left((\mathrm{~d} R) R^{-1}\right)=\left(\mathrm{d}[\mathbf{t} \mathbf{v} \mathbf{n}]^{\mathrm{T}}\right)[\mathbf{t} \mathbf{v} \mathbf{n}]=\left[\begin{array}{ccc}
0 & \mathbf{v} \cdot \mathrm{d} \mathbf{t} & \mathbf{n} \cdot \mathrm{d} \mathbf{t} \\
-\mathbf{v} \cdot \mathrm{d} \mathbf{t} & 0 & \mathbf{n} \cdot \mathrm{d} \mathbf{v} \\
-\mathbf{n} \cdot \mathrm{d} \mathbf{t} & -\mathbf{n} \cdot \mathrm{d} \mathbf{v} & 0
\end{array}\right] .
$$

According to Theorem 4.7 (and Remark 4.8), the one-forms (14) and (15) constitute a constant-structure $\mathrm{SO}(3)$-invariant coframe field on $J^{1}\left(\mathbb{R}^{2}, \mathbb{R}^{3}\right)$. Therefore, by Theorem 4.3, two surface immersions in $\mathbb{R}^{3}$ will be congruent under the action of $\mathrm{SO}(3)$ if and only if there exists a transformation $R \in \mathrm{SO}(3)$ taking some first-order jet $\left(\widetilde{x}, \widetilde{u}, \widetilde{u_{1}}, \widetilde{u_{2}}\right) \in J^{1}\left(\mathbb{R}^{2}, \mathbb{R}^{3}\right)$ of one of the surfaces to a jet $\left(\widetilde{x}, R \widetilde{u}, R \widetilde{u_{1}}, R \widetilde{u_{2}}\right)$ of the other surface and also evaluation of the coframe field (14) and (15) results in the same one-form system for both surfaces.

To shed a bit of light on the relationship of this result with the classic case of the full orientation-preserving rigid motion group $\mathrm{SE}(3)=\mathrm{SO}(3) \ltimes \mathbb{R}^{3}$, suppose that all the congruence conditions are met and thus the prolonged graphs of the two surfaces are congruent over an open subset of their domain $\mathbb{R}^{2}$. If the vector fields $u, u_{1}, u_{2}$ for each of the surfaces are expressed in terms of the orthonormal frame field $\{\mathbf{t}, \mathbf{v}, \mathbf{n}\}$ of the corresponding surface, the common restricted Maurer-Cartan forms (15) make the terms $[\mathrm{d} \mathbf{t} \mathrm{d} \mathbf{v} \mathrm{d} \mathbf{n}]^{\mathrm{T}} u,[\mathrm{~d} \mathbf{t} \mathrm{d} \mathbf{v} \mathrm{d} \mathbf{n}]^{\mathrm{T}} u_{1}$, $\left[\begin{array}{lll}\mathrm{d} \mathbf{d} & \mathrm{d} \mathbf{v} \mathrm{d} \mathbf{n}\end{array}\right]^{\mathrm{T}} u_{2}$ in (14) equal for both surfaces, and thus, in particular, the surfaces must agree on the terms

$$
\left[\begin{array}{lll}
\mathbf{t} & \mathbf{v} & \mathbf{n}
\end{array}\right]^{\mathrm{T}} \mathrm{d} u, \quad\left[\begin{array}{lll}
\mathbf{t} & \mathbf{v} & \mathbf{n}
\end{array}\right]^{\mathrm{T}} \mathrm{d} u_{1}, \quad\left[\begin{array}{lll}
\mathbf{t} & \mathbf{v} & \mathbf{n}
\end{array}\right]^{\mathrm{T}} \mathrm{d} u_{2} .
$$

Notice that specifying these one-forms (16) is equivalent (modulo some redundancy) to determining the first and second fundamental forms

$$
\mathrm{I}=(\mathbf{t} \cdot \mathrm{d} u) \otimes(\mathbf{t} \cdot \mathrm{d} u)+(\mathbf{v} \cdot \mathrm{d} u) \otimes(\mathbf{v} \cdot \mathrm{d} u), \quad \mathrm{II}=\sum_{i, j=1}^{2}\left(\mathbf{n} \cdot u_{i j}\right) \mathrm{d} x^{i} \otimes \mathrm{d} x^{j}
$$

that are classically known to form a complete system of congruence invariants for surface immersions under the transitive action of SE(3). Obviously, in view of the explanation given in the previous paragraph, the fundamental form conditions alone will not be sufficient for congruence under the intransitive action of our group $\mathrm{SO}(3)$.

A similar example is discussed in [3] that finds differential invariants of surfaces in $\mathbb{R}^{3}$ under the action of $\mathrm{SO}(3)$, but their invariants are for the variable-parameter congruence, as opposed to our fixed-parameter congruence invariants, and hence in particular do not include the first fundamental form. 


\subsection{Another nonhomogeneous space}

Lest we give the wrong impression through the preceding examples that our method is somehow confined to work only for well-known group actions, now we discuss for our final example ${ }^{5}$ a rather randomly chosen intransitive action on $\mathbb{R}^{3}$ and find a corresponding complete system of congruence invariants for surface immersions.

Let us consider the following action of a group $G$, parametrized by $\left(t_{1}, t_{2}, t_{3}, t_{4}, t_{5}\right)$, on $\mathbb{R}^{3}$ with coordinate system $(u, v, w)$ :

$$
G \times \mathbb{R}^{3} \longrightarrow \mathbb{R}^{3}, \quad\left(t_{1}, t_{2}, t_{3}, t_{4}, t_{5}\right) \cdot(u, v, w) \longmapsto\left(u+t_{1} v+t_{2} w+t_{3}, v+t_{4} w+t_{5}, w\right) .
$$

(This action can be viewed as one by a certain subgroup of a Heisenberg group as we will see later.)

For the first step to find a complete system of congruence invariants for surface immersions $\psi: \mathbb{R}^{2} \longrightarrow \mathbb{R}^{3}$ under the action of $G$, we verticalize the given action on $\mathbb{R}^{3}$ into one on the total space of the product bundle $\mathbb{R}^{2} \times \mathbb{R}^{3} \longrightarrow \mathbb{R}^{2}$ as follows:

$$
\begin{aligned}
& G \times\left(\mathbb{R}^{2} \times \mathbb{R}^{3}\right) \longrightarrow \mathbb{R}^{2} \times \mathbb{R}^{3}, \\
& \left(t_{1}, t_{2}, t_{3}, t_{4}, t_{5}\right) \cdot(x, y ; u, v, w) \longmapsto\left(x, y ; u+t_{1} v+t_{2} w+t_{3}, v+t_{4} w+t_{5}, w\right),
\end{aligned}
$$

where $(x, y)$ represents the coordinate system of $\mathbb{R}^{2}$, the domain of surface immersions. This verticalized $G$-action on $\mathbb{R}^{2} \times \mathbb{R}^{3}$ is not locally free, but its first-order prolongation

$$
\begin{aligned}
\mathcal{A}: & G \times J^{1}\left(\mathbb{R}^{2}, \mathbb{R}^{3}\right) \longrightarrow J^{1}\left(\mathbb{R}^{2}, \mathbb{R}^{3}\right), \\
& \left(t_{1}, t_{2}, t_{3}, t_{4}, t_{5}\right) \cdot\left(x, y ; u, v, w ; u_{x}, u_{y}, v_{x}, v_{y}, w_{x}, w_{y}\right) \\
& \longmapsto\left(x, y ; u+t_{1} v+t_{2} w+t_{3}, v+t_{4} w+t_{5}, w ;\right. \\
& \left.\quad u_{x}+t_{1} v_{x}+t_{2} w_{x}, u_{y}+t_{1} v_{y}+t_{2} w_{y}, v_{x}+t_{4} w_{x}, v_{y}+t_{4} w_{y}, w_{x}, w_{y}\right),
\end{aligned}
$$

is locally free since we can solve the following chosen normalization equations

$$
\begin{array}{ll}
\mathcal{A}^{*} u=u+t_{1} v+t_{2} w+t_{3}=0, & \mathcal{A}^{*} v=v+t_{4} w+t_{5}=0, \\
\mathcal{A}^{*} u_{x}=u_{x}+t_{1} v_{x}+t_{2} w_{x}=0, & \mathcal{A}^{*} u_{y}=u_{y}+t_{1} v_{y}+t_{2} w_{y}=0, \\
\mathcal{A}^{*} v_{x}=v_{x}+t_{4} w_{x}=0 &
\end{array}
$$

for the group parameters

$$
\begin{aligned}
& t_{1}=\frac{-u_{y} w_{x}+u_{x} w_{y}}{v_{y} w_{x}-v_{x} w_{y}}, \quad t_{2}=\frac{u_{y} v_{x}-u_{x} v_{y}}{v_{y} w_{x}-v_{x} w_{y}} \\
& t_{3}=\frac{-u v_{y} w_{x}-u_{y} w v_{x}+u_{y} v w_{x}+u v_{x} w_{y}+u_{x} w v_{y}-u_{x} v w_{y}}{v_{y} w_{x}-v_{x} w_{y}}, \\
& t_{4}=-\frac{v_{x}}{w_{x}}, \quad t_{5}=-v+\frac{w z_{x}}{w_{x}} .
\end{aligned}
$$

These solutions for $t_{1}, t_{2}, t_{3}, t_{4}, t_{5}$ are the ones used in the construction of the moving frame

$$
\rho: J^{1}\left(\mathbb{R}^{2}, \mathbb{R}^{3}\right) \longrightarrow G, \quad\left(x, y ; u, v, w ; u_{x}, u_{y}, v_{x}, v_{y}, w_{x}, w_{y}\right) \longmapsto\left(t_{1}, t_{2}, t_{3}, t_{4}, t_{5}\right),
$$

and its associated moving frame section $\sigma: J^{1}\left(\mathbb{R}^{2}, \mathbb{R}^{3}\right) \longrightarrow G \times J^{1}\left(\mathbb{R}^{2}, \mathbb{R}^{3}\right)$.

For the next step of finding Maurer-Cartan forms of $G$, we regard the action of $G$ on $\mathbb{R}^{3}$ as the linear group consisting of the following elements

$$
g:=\left[\begin{array}{cccc}
1 & t_{1} & t_{2} & t_{3} \\
0 & 1 & t_{4} & t_{5} \\
0 & 0 & 1 & 0 \\
0 & 0 & 0 & 1
\end{array}\right] \in \mathrm{GL}(4, \mathbb{R}), \quad t_{i} \in \mathbb{R}, \quad i=1, \ldots, 5,
$$

\footnotetext{
${ }^{5}$ Suggested by an anonymous referee.
} 
acting on the hyperplane $\left\{\left[\begin{array}{llll}u & v & w & 1\end{array}\right]^{\mathrm{T}} \in \mathbb{R}^{4} \mid u, v, w \in \mathbb{R}\right\}$ via the usual matrix multiplication. Then the linearly independent entries of the $\mathfrak{g l}(4, \mathbb{R})$-valued Maurer-Cartan form $(\mathrm{d} g) g^{-1}$,

$$
\mu^{1}:=\mathrm{d} t_{1}, \quad \mu^{2}:=-t_{4} \mathrm{~d} t_{1}+\mathrm{d} t_{2}, \quad \mu^{3}:=-t_{5} \mathrm{~d} t_{1}+\mathrm{d} t_{3}, \quad \mu^{4}:=\mathrm{d} t_{4}, \quad \mu^{5}:=\mathrm{d} t_{5},
$$

give rise to a Maurer-Cartan coframe field of $G$.

Now we use the formula (6) to construct constant-structure invariant coframe field of $J^{1}\left(\mathbb{R}^{2}, \mathbb{R}^{3}\right)$. Pulling back the Maurer-Cartan forms of $G$ by the moving frame $\rho$ yields

$$
\begin{aligned}
\rho^{*} \mu^{1}= & \frac{1}{\left(v_{y} w_{x}-v_{x} w_{y}\right)^{2}}\left(\left(v_{y} w_{x} w_{y}-v_{x} w_{y}^{2}\right) \mathrm{d} u_{x}+\left(-v_{y} w_{x}^{2}+v_{x} w_{x} w_{y}\right) \mathrm{d} u_{y}\right. \\
& +\left(-u_{y} w_{x} w_{y}+u_{x} w_{y}^{2}\right) \mathrm{d} v_{x}+\left(u_{y} w_{x}^{2}-u_{x} w_{x} w_{y}\right) \mathrm{d} v_{y}+\left(u_{y} v_{x} w_{y}-u_{x} v_{y} w_{y}\right) \mathrm{d} w_{x} \\
& \left.+\left(-u_{y} v_{x} w_{x}+u_{x} v_{y} w_{x}\right) \mathrm{d} w_{y}\right), \\
\rho^{*} \mu^{2}= & \frac{1}{v_{y} w_{x}^{2}-v_{x} w_{x} w_{y}}\left(\left(-v_{y} w_{x}+v_{x} w_{y}\right) \mathrm{d} u_{x}+\left(u_{y} w_{x}-u_{x} w_{y}\right) \mathrm{d} v_{x}+\left(-u_{y} v_{x}+u_{x} v_{y}\right) \mathrm{d} w_{x}\right), \\
\rho^{*} \mu^{3}= & \frac{1}{v_{y} w_{x}^{2}-v_{x} w_{x} w_{y}}\left(\left(-v_{y} w_{x}^{2}+v_{x} w_{x} w_{y}\right) \mathrm{d} u+\left(u_{y} w_{x}^{2}-u_{x} w_{x} w_{y}\right) \mathrm{d} v\right. \\
& +\left(w v_{y} w_{x}-w v_{x} w_{y}\right) \mathrm{d} u_{x}+\left(-w u_{y} w_{x}+w u_{x} w_{y}\right) \mathrm{d} v_{x}+\left(-u_{y} v_{x} w_{x}+u_{x} v_{y} w_{x}\right) \mathrm{d} w \\
& \left.+\left(w u_{y} v_{x}-w u_{x} v_{y}\right) \mathrm{d} w_{x}\right), \\
\rho^{*} \mu^{4}= & -\frac{1}{w_{x}} \mathrm{~d} v_{x}+\frac{v_{x}}{w_{x}^{2}} \mathrm{~d} w_{x}, \\
\rho^{*} \mu^{5}= & -\mathrm{d} v+\frac{v_{x}}{w_{x}} \mathrm{~d} w+\frac{w}{w_{x}} \mathrm{~d} v_{x}-\frac{w v_{x}}{w_{x}^{2}} \mathrm{~d} w_{x},
\end{aligned}
$$

and pulling back the coordinate functions of $J^{1}\left(\mathbb{R}^{2}, \mathbb{R}^{3}\right)$, that were not normalized to constants, by $\iota=\mathcal{A} \circ \sigma$ and then taking their exterior differentials yields

$$
\begin{aligned}
& \mathrm{d} \iota^{*} x=\mathrm{d} x, \quad \mathrm{~d} \iota^{*} y=\mathrm{d} y, \quad \mathrm{~d} \iota^{*} w=\mathrm{d} w, \\
& \mathrm{~d} \iota^{*} v_{y}=-\frac{w_{y}}{w_{x}} \mathrm{~d} v_{x}+\mathrm{d} v_{y}+\frac{v_{x} w_{y}}{w_{x}^{2}} \mathrm{~d} w_{x}-\frac{v_{x}}{w_{x}} \mathrm{~d} w_{y}, \\
& \mathrm{~d} \iota^{*} w_{x}=\mathrm{d} w_{x}, \quad \mathrm{~d} \iota^{*} w_{y}=\mathrm{d} w_{y} .
\end{aligned}
$$

The eleven one-forms, (17) and (18), constitute a constant-structure $G$-invariant coframe field of $J^{1}\left(\mathbb{R}^{2}, \mathbb{R}^{3}\right)$. The final step now is to pull back those coframe one-forms by the prolonged graph $j^{1} \gamma(\psi): \mathbb{R}^{2} \longrightarrow J^{1}\left(\mathbb{R}^{2}, \mathbb{R}^{3}\right)$ of a generic surface $\psi: \mathbb{R}^{2} \longrightarrow \mathbb{R}^{3},(x, y) \longmapsto(u(x, y), v(x, y), w(x, y))$, to obtain the following system of one-forms:

$$
\begin{aligned}
\left(j^{1} \gamma(\psi)\right)^{*} \rho^{*} \mu^{1}= & \frac{\mathrm{d} x}{\left(v_{y} w_{x}-v_{x} w_{y}\right)^{2}}\left(-u_{y} v_{x x} w_{x} w_{y}+u_{x x} v_{y} w_{x} w_{y}-u_{x x} v_{x} w_{y}^{2}\right. \\
& +u_{x} v_{x x} w_{y}^{2}+u_{y} v_{x} w_{x x} w_{y}-u_{x} v_{y} w_{x x} w_{y}-v_{y} w_{x}^{2} u_{x y}+u_{y} w_{x}^{2} v_{x y} \\
& \left.+v_{x} w_{x} w_{y} u_{x y}-u_{x} w_{x} w_{y} v_{x y}-u_{y} v_{x} w_{x} w_{x y}+u_{x} v_{y} w_{x} w_{x y}\right) \\
& +\frac{\mathrm{d} y}{\left(v_{y} w_{x}-v_{x} w_{y}\right)^{2}}\left(-u_{y y} v_{y} w_{x}^{2}+u_{y} v_{y y} w_{x}^{2}+u_{y y} v_{x} w_{x} w_{y}-u_{x} v_{y y} w_{x} w_{y}\right. \\
& -u_{y} v_{x} w_{x} w_{y y}+u_{x} v_{y} w_{x} w_{y y}+v_{y} w_{x} w_{y} u_{x y}-u_{y} w_{x} w_{y} v_{x y}-v_{x} w_{y}^{2} u_{x y} \\
& \left.+u_{x} w_{y}^{2} v_{x y}+u_{y} v_{x} w_{y} w_{x y}-u_{x} v_{y} w_{y} w_{x y}\right), \\
\left(j^{1} \gamma(\psi)\right)^{*} \rho^{*} \mu^{2}= & \frac{\mathrm{d} x}{v_{y} w_{x}^{2}-v_{x} w_{x} w_{y}}\left(u_{y} v_{x x} w_{x}-u_{x x} v_{y} w_{x}-u_{y} v_{x} w_{x x}+u_{x} v_{y} w_{x x}\right.
\end{aligned}
$$




$$
\begin{aligned}
& \left.+u_{x x} v_{x} w_{y}-u_{x} v_{x x} w_{y}\right)+\frac{\mathrm{d} y}{v_{y} w_{x}^{2}-v_{x} w_{x} w_{y}}\left(-v_{y} w_{x} u_{x y}+u_{y} w_{x} v_{x y}\right. \\
& \left.+v_{x} w_{y} u_{x y}-u_{x} w_{y} v_{x y}-u_{y} v_{x} w_{x y}+u_{x} v_{y} w_{x y}\right) \\
\left(j^{1} \gamma(\psi)\right)^{*} \rho^{*} \mu^{3}= & \frac{\mathrm{d} x}{v_{y} w_{x}^{2}-v_{x} w_{x} w_{y}}\left(-w u_{y} v_{x x} w_{x}+w u_{x x} v_{y} w_{x}+w u_{y} v_{x} w_{x x}\right. \\
& \left.-w u_{x} v_{y} w_{x x}-w u_{x x} v_{x} w_{y}+w u_{x} v_{x x} w_{y}\right)+\frac{\mathrm{d} y}{v_{y} w_{x}^{2}-v_{x} w_{x} w_{y}}\left(w v_{y} w_{x} u_{x y}\right. \\
& \left.-w u_{y} w_{x} v_{x y}-w v_{x} w_{y} u_{x y}+w u_{x} w_{y} v_{x y}+w u_{y} v_{x} w_{x y}-w u_{x} v_{y} w_{x y}\right), \\
\left(j^{1} \gamma(\psi)\right)^{*} \rho^{*} \mu^{4}= & \left(\frac{v_{x} w_{x x}}{w_{x}^{2}}-\frac{v_{x x}}{w_{x}}\right) \mathrm{d} x+\left(\frac{v_{x} w_{x y}}{w_{x}^{2}}-\frac{v_{x y}}{w_{x}}\right) \mathrm{d} y \\
\left(j^{1} \gamma(\psi)\right)^{*} \rho^{*} \mu^{5}= & \left(\frac{w v_{x x}}{w_{x}}-\frac{w v_{x} w_{x x}}{w_{x}^{2}}\right) \mathrm{d} x+\left(\frac{v_{x} w_{y}}{w_{x}}-\frac{w v_{x} w_{x y}}{w_{x}^{2}}+\frac{w v_{x y}}{w_{x}}-v_{y}\right) \mathrm{d} y, \\
\left(j^{1} \gamma(\psi)\right)^{*} \mathrm{~d} \iota^{*} x= & \mathrm{d} x, \\
\left(j^{1} \gamma(\psi)\right)^{*} \mathrm{~d} \iota^{*} y= & \mathrm{d} y, \\
\left(j^{1} \gamma(\psi)\right)^{*} \mathrm{~d} \iota^{*} w= & w_{x} \mathrm{~d} x+w_{y} \mathrm{~d} y, \\
\left(j^{1} \gamma(\psi)\right)^{*} \mathrm{~d} \iota^{*} v_{y}= & \left(\frac{v_{x} w_{x x} w_{y}}{w_{x}^{2}}-\frac{v_{x x} w_{y}}{w_{x}}-\frac{v_{x} w_{x y}}{w_{x}}+v_{x y}\right) \mathrm{d} x+\left(-\frac{v_{x} w_{y y}}{w_{x}}\right. \\
& \left.-\frac{w_{y} v_{x y}}{w_{x}}+\frac{v_{x} w_{y} w_{x y}}{w_{x}^{2}}+v_{y y}\right) \mathrm{d} y
\end{aligned}
$$

Thus, a complete system of $G$-congruence invariants for surface immersions in $\mathbb{R}^{3}$ is provided by the set of all the coefficients of $\mathrm{d} x$ and $\mathrm{d} y$ in (19). According to Theorem 4.3, two surface immersions will be congruent under the action of $G$ if and only if some transformation $g \in G$ takes some first-order jet of one of the surfaces to a jet of the other surface and the invariants (19) evaluated for the two surfaces agree.

\section{Discussions}

We introduced the notion of invariant coframe fields of constant structure, whose explicit construction can be done by the method of equivariant moving frames, and used them to prove Theorem 4.3 that provided theoretical justification of our coherent method of completely solving the congruence problem of immersions in homogeneous and nonhomogeneous spaces alike. It extends and generalizes to arbitrary $G$-spaces the key congruence lemmas in [5] that were designed for homogeneous spaces. We demonstrated our method by applying it to congruence problems in some classical and other examples.

The next order of research in this direction should involve applications of our method to more substantial and unexplored congruence problems.

\section{Acknowledgments}

This work has benefited from the discussions held in the Differential Geometry and Lie Theory seminars at the University of Toledo; the author would like to thank the organizers and participants of the seminars. Also, the anonymous referees' critical and yet helpful comments have contributed significantly in the process of revising and improving the paper; the author is very grateful to the referees. 
It is hoped that this work serves to reflect, although only to a small extent limited by the author's meager knowledge, the author's appreciation of the introduction by Professor Peter Olver to the marvelous unifying philosophy and technology of symmetry, invariance, and equivalence.

\section{References}

[1] Anderson I.M., Introduction to the variational bicomplex, in Mathematical Aspects of Classical Field Theory (Seattle, WA, 1991), Contemp. Math., Vol. 132, Amer. Math. Soc., Providence, RI, 1992, 51-73.

[2] Fels M., Olver P.J., Moving coframes. I. A practical algorithm, Acta Appl. Math. 51 (1998), 161-213.

[3] Fels M., Olver P.J., Moving coframes. II. Regularization and theoretical foundations, Acta Appl. Math. 55 (1999), 127-208.

[4] Green M.L., The moving frame, differential invariants and rigidity theorems for curves in homogeneous spaces, Duke Math. J. 45 (1978), 735-779.

[5] Griffiths P., On Cartan's method of Lie groups and moving frames as applied to uniqueness and existence questions in differential geometry, Duke Math. J. 41 (1974), 775-814.

[6] Hubert E., Olver P.J., Differential invariants of conformal and projective surfaces, SIGMA 3 (2007), 097, 15 pages, arXiv:0710.0519.

[7] Ivey T.A., Landsberg J.M., Cartan for beginners: differential geometry via moving frames and exterior differential systems, Graduate Studies in Mathematics, Vol. 61, Amer. Math. Soc., Providence, RI, 2003.

[8] Kogan I.A., Olver P.J., Invariant Euler-Lagrange equations and the invariant variational bicomplex, Acta Appl. Math. 76 (2003), 137-193.

[9] Olver P.J., Applications of Lie groups to differential equations, 2nd ed., Graduate Texts in Mathematics, Vol. 107, Springer-Verlag, New York, 1993.

[10] Olver P.J., Differential invariants of surfaces, Differential Geom. Appl. 27 (2009), 230-239.

[11] Olver P.J., Equivalence, invariants, and symmetry, Cambridge University Press, Cambridge, 1995.

[12] Olver P.J., Moving frames and differential invariants in centro-affine geometry, Lobachevskii J. Math. 31 (2010), 77-89.

[13] Olver P.J., Pohjanpelto J., Differential invariant algebras of Lie pseudo-groups, Adv. Math. 222 (2009), 1746-1792.

[14] Olver P.J., Pohjanpelto J., Moving frames for Lie pseudo-groups, Canad. J. Math. 60 (2008), $1336-1386$.

[15] Sternberg S., Lectures on differential geometry, 2nd ed., Chelsea Publishing Co., New York, 1983.

[16] Warner F.W., Foundations of differentiable manifolds and Lie groups, Graduate Texts in Mathematics, Vol. 94, Springer-Verlag, New York, 1983. 